\title{
GEODESIC FLOWS ON NEGATIVELY CURVED MANIFOLDS. II $\left.{ }^{1}\right)$
}

\author{
BY
}

\section{PATRICK EBERLEIN}

\begin{abstract}
Let $M$ be a complete Riemannian manifold with sectional curvature $K \leq 0, S M$ the unit tangent bundle of $M, T$ the geodesic flow on $S M$ and $\Omega \subseteq S M$ the set of nonwandering points relative to $T_{t} . T_{t}$ is topologically mixing (respectively topologically transitive) on $S M$ if for any open sets $O, U$ of $S M$ there exists $A>0$ such that $|t| \geq A$ implies $T_{t}(O) \cap U \neq$ $\varnothing$ (respectively there exists $t \in R$ such that $\left.T_{t}(()) \cap U \neq \varnothing\right)$. For each vector $v \in S M$ we define stable and unstable sets $W s(v), W s s(v), W u(v)$ and $W u u(v)$, and we relate topological mixing (respectively topological transitivity) of $T_{t}$ to the existence of a vector $v \in S M$ such that WSS( $v$ ) (respectively $\left.W^{S}(v)\right)$ is dense in $S M$. If $M$ is a Visibility manifold (implied by $K \leq c<0$ ) and if $\boldsymbol{\Omega}=S M$ then $T_{t}$ is topologically mixing on $S M$. I.et $S_{n}=\{$ Visibility manifolds $M$ of dimension $n$ such that $T_{t}$ is topologically mixing on $\left.S M\right\}$. For each $n \geq 2, S_{n}$ is closed under normal (Galois) Riemannian coverings. If $M \in S_{n}$ we classify $\{l \in S M: W s s(v)$ is dense in $S M\}$, and $M$ is compact if and only if this set $S M$. We also consider the case where $\Omega$ is a proper subset of $S M$.
\end{abstract}

Introduction. Let $H$ be any complete, simply connected Riemannian manifold with sectional curvature $K \leq 0$. Each equivalence class $x$ of asymptotic geodesics in $H$ determines a family of parallel limit spheres with center $x$. Unit vectors $v, w$ tangent to $H$ are weakly asymptotic if the geodesics $\gamma_{v}$ and $\gamma_{w}$ that they determine are asymptotic. $v$ and $w$ are strongly asymptotic if, in addition, their points of tangency lie on the same limit sphere determined by the equivalence class of $\gamma_{v}$ and $\gamma_{w}$. $"$ and $w$ are weakly (strongly) unstably asymptotic if $-v$ and $-w$ are weakly (strongly) asymptotic. Let $W^{s}(v), W^{s s}(v)$, $W^{u}(v), W^{u u}(v)$ be the collections of weak, strong, weak unstable and strong unstable asymptotes of $\nu$. These sets will be called the stable, strong stable, unstable and strong unstable sets determined by $\nu$. A complete manifold $M$ with $K \leq 0$ may be represented as a quotient $H / D$ where $D$ is a properly discontinuous group of isometries of $H$. If $M=H / D$ we define the stable and unstable

Received by the editors August 2, 1971.

AMS (MOS) subject classifications (1970). Primary 53A35, 53C.20; Secondary 28A65, 34C 35, 58F99.

Key uords and phrases. Geodesic flow, prolongational limit sets, nonwandering points, stable and unstable sets, topological transitivity, topological mixing, Axiom 1, Visibility manifold.

(1) This research was supported in part by NSF Grants GP.11476 and GP.20096. 
sets in the unit tangent bundle of $M$ by projection; if $\pi: H \rightarrow M$ is the projection, then $W^{s s}\left(\pi_{*} v\right)=\pi_{*} W^{s s}(v)$ for any unit vector $v$ tangent to $H$ and similarly for the other sets.

The sets $W^{s}, W^{s s}, W^{u}$ and $W^{u u}$ are defined for each unit vector $v$ tangent to an arbitrary manifold $M$ with $K \leq 0$ and coincide with the usual stable and unstable manifolds if $M$ is compact with $K<0$. These sets (primarily $W^{s s}$ ) were first studied as horocycles by Hedlund [12] and Grant [9] and applied to the geodesic flow of surfaces of constant negative curvature and certain kinds of variable negative curvature. Horocycles were later generalized to stable and unstable manifolds, which are defined for flows more general than the geodesic flow on a compact manifold with $K<0$. The generalization of horocycles to the stable and unstable sets defined here appears to be a natural way to study the geodesic flow on an arbitrary complete manifold $M$ with $K \leq 0$. The relationship of these sets (which possibly are not manifolds) to the geodesic flow appears clearly in $\$ 4$, especially in Propositions 4.6 and 4.11 .

The connection between the sets $W^{s}$ and $W^{s s}$ and the properties of topological transitivity and topological mixing occurs in Theorems 4.14, 4.15 and 4.16. In Theorem 4.15 we allow $\Omega$ to be any subset of the unit tangent bundle of $M$. We considered in [6] those manifolds $M$ without conjugate points whose simply connected Riemannian covering $H$ satisfies the uniform Visibility axiom, and we showed that if $\Omega=S M$, the unit tangent bundle of $M$, then the geodesic flow is topologically transitive on $S M$. At the end of $\$ 4$ we indicate the difficulties involved in proving the topological mixing of the geodesic flow on $S M$ by the method used here.

$\S 5$ contains results on the existence and classification of vectors $v \in \Omega$ such that $\Omega \subseteq \overline{W^{s s}(v)}$. The main applications and results are in $\$ \sigma$. We note that the geodesic flow is topologically mixing for certain manifolds $M=H / D$ with some zero curvature (Theorem 6.4) and for many noncompact manifolds $M$ with infinite volume (Theorem 6.5).

The stable and unstable sets offer a promising way to investigate ergodic and metrical mixing problems of the geodesic flow on $S M$, where $M$ is any complete manifold with $K \leq 0$. For example, we define a set $A C S M$ to be $W^{s}$ invariant if $W^{s}(v) \subset A$ whenever $v \in A$. Is the geodesic flow in $S M$ ergodic if it is true that, for any $W^{S}$ invariant set $A$, either $A$ or $S M-A$ has measure zero relative to the natural flow invariant measure on $S M$ ? Is the geodesic flow in $S M$ metrically mixing if the corresponding property for $W^{s s}$ invariant sets $A C$ SM holds? Questions of this sort should be answered.

1. Notation and preliminaries. For any Riemannian manifold $M$ we denote the Riemannian structure by $\langle$,$\rangle , the Riemannian metric by d$ and the sectional 
curvature by $K$. If $p \in M$ let $S(p)$ be the unit sphere in the tangent space $M_{p}$, and let $S M$ be the unit tangent bundle of $M$ with the projection $\mu: S M \rightarrow M$. If $v, w \in S(p)$ the angle $\theta=\not \backslash(\nu, w)$ is the unique number $0 \leq \theta \leq \pi$ such that $\langle v, w\rangle=\cos \theta$. If $M$ is complete and $v \in S M$, let $\gamma_{v}: R \rightarrow M$ be the geodesic such that $\gamma_{v}^{\prime}(0)=v$. We shall assume that all geodesics bave unit speed and are defined on the entire real line unless otherwise indicated. A geodesic segment is a geodesic defined on a compact interval.

A Hadamard manifold $H$ is a complete, simply connected Riemannian manifold of dimension $n \geq 2$ with curvature $K \leq 0$. $H$ will always denote a Hadamard manifold. If $p \neq q$ are points in $H$, let $\gamma_{p q}$ be the unique geodesic such that $\gamma_{p q}(0)=p$ and $\gamma_{p q}(t)=q$ where $t=d(p, q)$. The angle $\chi_{p}(m, n)$ subtended at $p$ by points $m, n$ of $H$ distinct from $p$ is $\not\left\langle\left(\gamma_{p m}^{\prime}(0), \gamma_{p n}^{\prime}(0)\right)\right.$.

The complete manifolds $M$ with curvature $K \leq 0$ are the quotient manifolds $H / D$ where $H$ is a Hadamard manifold and $D$ is a properly discontinous group of isometries of $H$. D will always denote such a group; $\pi: H \rightarrow M=H / D$ will always denote the projection and $\pi_{*}: S H \rightarrow S M$ the differential map of $\pi$.

We list some basic definitions and results (mostly without proofs) that are discussed in great detail with proofs in $\$ 1$ and $\$ 2$ of [8]. See also [7].

Definition 1.1. Geodesics $\alpha$ and $\beta$ in $H$ are asymptotic if there exists a number $c>0$ such that $d(\alpha t, \beta t) \leq c$ for all $t \geq 0$. Geodesics $\gamma, \sigma$ in $H / D$ are asymptotic if they have lifts $\alpha, \beta$ in $H$ that are asymptotic.

The asymptote relation is an equivalence relation on the set geodesics in $H$ and in $H^{\prime} D$. A point at infinity for $H$ is an equivalence class of asymptotic geodesics of $H$. For any geodesic $\gamma$ in $H$ let $\gamma(\infty)$ denote the asymptote equivalence class of $\gamma$ and $\gamma(-\infty)$ the class of the reverse curve $t \rightarrow \gamma(-t)$. Note that $\gamma(\infty) \neq \gamma(-\infty)$ since $\gamma$ realizes the distance between any two of its points. Let $H(\infty)$ be the set of points at infinity for $H$, and let $\bar{H}=H \cup H(\infty)$. For any point $p \in H$ let $B_{p}$ be the closed unit ball in $H_{p}$; we define a bijection $F_{p}: B_{p} \rightarrow \bar{H}$ and $F_{p}(S(p))=H(\infty)$. Let $F_{p}(v)=\exp _{p}(\nu / 1-\|v\|)$ if $\|\nu\|<1$ and let $F_{p}(v)=\gamma_{v}(\infty)$ if $\|v\|=1$. For each $p$ there is a unique topology on $\bar{H}$ such that $F_{p}$ is a homeomorphism. These topologies coincide for each $p$ with the cone topology defined invariantly in $\$ 2$ of [8], and $H$ is a dense open subset of $\bar{H}$. We shall later define the borocycle topology for $\bar{H}$, but in the sequel we shall always mean the cone topology if the topology of $\bar{H}$ is unspecified.

If $p \in H$ and $x \in H(\infty)$, there is a unique geodesic $\gamma_{p x}$ such that $\gamma_{p x}(0)=$ $p$ and $\gamma_{p x}(\infty)=x$. If $p \in H$ is distinct from points $a, b$ in $\bar{H}$, then the angle $X_{p}(a, b)$ subtended at $p$ by $a$ and $b$ is $\not \subset\left(\gamma_{p a}^{\prime}(0), \gamma_{p b}^{\prime}(0)\right)$. If $S \subseteq \bar{H}$ is any set not containing $p \in H$, then the angle $\chi_{p}(S)$ subtended at $p$ by $S$ is 
$\sup \left\{\not_{p}(a, b): a \in S, b \in S\right\}$. The following results are basic and natural.

Proposition 1.2. Let $A=\{(p, b) \in H \times \bar{H}: p \neq b\}$. The vector function $V: A \rightarrow S H$ given by $V(p, b)=\gamma_{p b}^{\prime}(0)$ is continuous, and $V \mid H \times H(\infty)$ is a bomeomorphism onto $\mathrm{SH}$.

Proposition 1.3. The angle function $\chi_{p}(a, b)$ is continuous on $\{(p, a, b) \in$ $H \times \bar{H} \times \bar{H}: p \neq a, p \neq b\}$.

Proposition 1.4. The function $\psi: S H \times[-\infty, \infty] \rightarrow \bar{H}$ given by $\psi(v, t)=\gamma_{v}(t)$ is continuous.

It follows from Propositions 1.2 and 1.3 that if $x \in H(\infty)$ and $p_{n} \subseteq \bar{H}$ is a sequence, then $p_{n} \rightarrow x$ if and only if $\chi_{p}\left(p_{n}, x\right) \rightarrow 0$ for any point $p \in H$.

If $\phi$ is an isometry of $H$ and if $x$ is any point in $H(\infty)$ we define $\phi(x)=$ $(\phi \circ \alpha)(\infty)$ where $\alpha$ is any geodesic representing $x$. $\phi$ extends to a well-defined homeomorphism of $\bar{H}$. A properly discontinuous group $D$ of isometries of $H$ becomes a group of homeomorphisms of $\bar{H}$. The limit set $L(D)$ of $D$ is the set of accumulation points in $H(\infty)$ of an orbit $D(p)$, where $p$ is any point of $H$. By the corollary of the law of cosines immediately following Proposition 2.1 in $\$ 2$, $L(D)$ does not depend on the point $p$. If $D$ is not the identity, then $L(D)$ is a nonempty closed subset of $H(\infty)$ that is invariant under $D$.

If $x$ and $y$ are distinct points in $H(\infty)$, a geodesic $a$ of $H$ is said to join $x$ to $y$ if $a(-\infty)=x$ and $a(\infty)=y$. $H$ satisfies Axiom 1 (Axiom 2) if for any points $x \neq y$ in $H(\infty)$ there exists at least (at most) one geodesic joining $x$ to $y$. Both axioms are satisfied if the curvature of $H$ satisfies $K \leq c<0$. Axiom 1 is perhaps the most important single geometric property of such manifolds. There are several equivalent formulations of Axiom 1 and we mention two of the most useful ones.

Proposition 1.5. The following properties are equivalent in an arbitrary Hadamard manifold $H$ :

(1) H satisfies Axiom 1.

(2) Let $x$ be any point in $H(\infty), p_{n}$ a sequence in $H$ converging to $x$, and $W$ a neigbborbood of $x$ in $\bar{H}$. Then $\chi_{p_{n}}(\bar{H}-W) \rightarrow 0$.

(3) For every point $p \in H$ and every $\epsilon>0$ there exists a number $r=r(p, \epsilon)$ with this property: if $\sigma:[a, b] \rightarrow H$ is a geodesic such that $d(p, \sigma) \geq r$, then $\chi_{p}(\sigma a, \sigma b) \leq \epsilon$.

Property (3) is referred to as the Visibility axiom in [6], 17], and [8] since distant geodesics look small. If $r$ can be chosen to depend only on $\epsilon$, then we have the uniform Visibility axiom. 
Definition 1.6. $M=H / D$ is a (uniform) Visibility manifold if $H$ satisfies the (uniform) Visibility axiom.

Proof of Proposition 1.5. The assertions (3) $\Rightarrow(2)$ and (3) $\Rightarrow(1)$ are proved in [8] in Proposition 4.7 and the discussion following Proposition 4.4. Lemma 4.3 of [6] states that if property (3) fails in $H$, then there exist points $x \neq y$ in $H(\infty)$ such that $\chi_{p}(x, y) \leq \pi / 2$ for every point $p \in H$. The assertion (1) $\Rightarrow$ (3) now follows immediately. An independent proof of this fact may be found in [15]. To prove $(2) \Rightarrow(3)$ we need only show that for any two points $x \neq y$ in $H(\infty)$ there exists a point $p$ in $H$ such that $\Varangle_{p}(x, y)>\pi / 2$. Assume that (2) holds and let points $x \neq y$ be given. Let $\gamma$ be a geodesic in $H$ such that $\gamma(\infty)=x$, and let $W$ be a neighborhood of $x$ in $\bar{H}$ that excludes $z=\gamma(-\infty)$ and $y . \gamma(t) \rightarrow \gamma(\infty)$ as $t \rightarrow \infty$ by Proposition 1.4, and therefore by property (2), if $t$ is sufficiently large, then $\chi_{\gamma_{t}}(z, y)<\pi / 2$ and $\chi_{\gamma_{t}}(x, y)=\pi-\not_{\gamma_{t}}(z, y)>\pi / 2$.

Remark 1.7. Let $H$ sat isfy Axiom 1.

(1) If $r=r(p, \epsilon)$ as in property (3) and if $\alpha$ is a geodesic in $H$ such that $d(p, a) \geq r$ then $\chi_{p}(\alpha) \leq \epsilon$.

(2) If $\alpha_{n}$ is a sequence of geodesics in $H$ such that $d\left(p, \alpha_{n}\right) \rightarrow \infty$ then $\chi_{p}\left(\alpha_{n}\right) \rightarrow 0$.

2. Limit spheres and the horocycle topology. In this section we define and list some basic properties of limit spheres (horospheres). In dimension two these are classically referred to as horocycles and were first used systematically by Hedlund to study the geodesic flow on a compact surface of constant or nearly constant negative curvature. We extend his idea to arbitrary manifolds $M=H / D$. Our approach is modeled on that of Busemann [5].

Proofs of some assertions are omitted and may be found in $\$ 3$ of [8].

Our goal is to define "spheres" in $H$ whose "center" is a point $x \in H(\infty)$. These spheres should share many of the properties of finite spheres with centers in $H$. For example, a sphere should be uniquely determined by a point $p \in H$ and the center $x \in H(\infty)$, and it should vary continuously with the point $p$ and the center $x$. There should be some way to measure the relative proximity of points $p, q$ in $H$ to a point $x \in H(\infty)$. Points $p$ and $q$ should lie on the same sphere with center $x$ if they are "equidistant" from $x$. By adapting an idea of Busemann we make these vague notions precise by defining an essentially unique radius function for each point $x \in H(\infty)$ whose level sets are the spheres with center $x$. Busemann's radius function may be defined in any $G$-space although the spheres he obtains as level sets are not well behaved without some nice properties of the space. The law of cosines in a Hadamard manifold $H$ is a nice property and removes all difficulties.

The following result is useful. 
Proposition 2.1. If $A, B, C$ are three noncollinear points in $H$, then the geodesic triangle they determine with sides of length $a, b$, and $c$ and opposite angles $\alpha, \beta$ and $\gamma$ satisfies these relations:

(1) $c \leq b \cos \alpha+a \cos \beta$,

(2) $1-\cos \gamma \leq c^{2} / 2 a b$.

Proof. The law of cosines states that $c^{2} \geq a^{2}+b^{2}-2 a b \cos \gamma$. Thus $b^{2}$ $\geq a^{2}+c^{2}-2 a c \cos \beta \geq b^{2}+2 c^{2}-2 b c \cos \alpha-2 a c \cos \beta$. Simplifying we find that $0 \geq c-b \cos \alpha-a \cos \beta$ which proves (1). To prove (2) we note that $c^{2} \geq$ $a^{2}+b^{2}-2 a b \cos \gamma=(a-b)^{2}+2 a b(1-\cos \gamma) \geq 2 a b(1-\cos \gamma)$.

It follows from (2) above that if $p_{n}, q_{n}$ and $r_{n}$ are sequences in $H$ such that $d\left(p_{n}, q_{n}\right) \rightarrow \infty$ and $d\left(q_{n}, r_{n}\right)$ is bounded above, then $\chi_{p_{n}}\left(q_{n}, r_{n}\right) \rightarrow 0$.

Definition 2.2. Let $B: S H \times H \rightarrow R$ be defined by $B(\nu, p)=$ $\lim _{t \rightarrow+\infty} d\left(p, \gamma_{v} t\right)-t$.

$B$ is well defined since $t \rightarrow d\left(p, \gamma_{v} t\right)-t$ is monotone nonincreasing by the triangle inequality and bounded below by $-d\left(p, \gamma_{v} \overline{0}\right.$.

A function $f:[a, b] \rightarrow R$ is convex on $[a, b]$ if for any $0 \leq \lambda \leq 1$, $f(\lambda a+(1-\lambda) b) \leq \lambda f(a)+(1-\lambda) f(b) . f: H \rightarrow R$ is convex if for any geodesic $a$, $f \circ a: R \rightarrow R$ is convex.

Proposition 2.3. (1) For fixed $v \in S H$, the function $p \rightarrow B(v, p)$ is a uniformly continuous convex function on $H$ such that $|B(\nu, p)-B(v, q)| \leq d(p, q)$.

(2) $B: S H \times H \rightarrow R$ is continuous.

Proof. (1) The triangle inequality shows that $|B(v, p)-B(v, q)| \leq d(p, q)$ and uniform continuity follows from this inequality. Let a geodesic segment $a$ : $[a, b] \rightarrow H$ and $0 \leq \lambda \leq 1$ be given. Fix $s>0$. By Proposition 4.7 of [4] the function $p \rightarrow d\left(\gamma_{v} s, p\right)$ is a continuous convex function and hence $d\left(\gamma_{v} s, a(\lambda a+(1-\lambda) b)\right) \leq \lambda d\left(\gamma_{v} s, a a\right)+(1-\lambda) d\left(\gamma_{v} s, a b\right)$. Hence $d\left(\gamma_{v} s, a(\lambda a+(1-\lambda) b)\right)-s \leq \lambda\left\{d\left(\gamma_{v} s, a a\right)-s\right\}+(1-\lambda)\left\{d\left(\gamma_{v} s, a b\right)-s\right\}$. Letting $s-+\infty$ on both sides of the inequality we conclude that $B$ is convex.

(2) For each $t>0$ let $B_{t}: S H \times H \rightarrow R$ be given by $B_{t}(v, p)=d\left(p, \gamma_{v} t\right)-$ $t$. For each $t>0, B_{t}$ is continuous and $B_{t} \rightarrow B$ pointwise as $t \rightarrow+\infty$. To prove the continuity of $B$ it suffices to prove that for every compact set $C \subseteq$ $S H \times H$ and every $\epsilon>0$ there exists a number $T=T(C, \epsilon)$ such that, for $t \geq T$, $\left|B_{t}-B\right|<\epsilon$ on $C$. This result will follow from

Lemma 2.4. Let $T>0$ be given. If $s \geq T, t \geq T$ then $\left|B_{t}(\nu, p)-B_{s}(\nu, p)\right|$ $\leq d^{2}(p, \mu \nu) / T$ where $\mu: S H \rightarrow H$ is the natural projection. 
Proof.

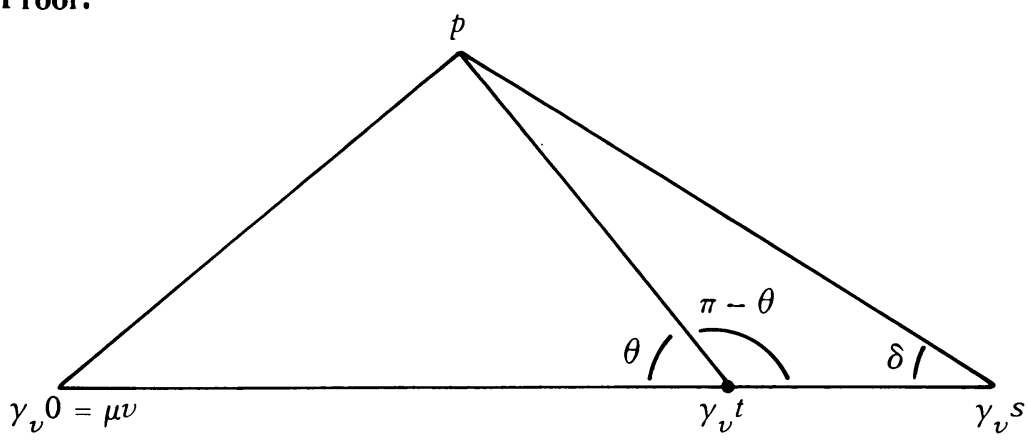

Figure 1

Let $s>t \geq T>0$ and $(\nu, p) \in S H \times H$ be given. Let $\theta=X_{\gamma_{v} t}(p, \mu v), \delta=$ $\chi_{\gamma_{\nu} s}(p, \mu v)$. Since $B_{t}$ is decreasing in $t, 0 \leq B_{t}(\nu, p)-B_{s}(v, p)=(s-t)+$ $d\left(p, \gamma_{v} t\right)-d\left(p, \gamma_{v} s\right)$. By Proposition 2.1 $(s-t) \leq d\left(p, \gamma_{v} t\right) \cos (\pi-\theta)+$ $d\left(p, \gamma_{v} s\right) \cos \delta$. Thus $B_{t}(\nu, p)-B_{s}(\nu, p) \leq d\left(p, \gamma_{\nu} t\right)(1-\cos \theta)+d\left(p, \gamma_{v} s\right)$ . $(1-\cos \delta)$. By Proposition $2.1(2), d\left(p, \gamma_{v} t\right)(1-\cos \theta) \leq d^{2}(p, \mu v) / 2 t \leq$ $d^{2}(p, \mu \nu) / 2 T$. Similarly $d\left(p, \gamma_{\nu} s\right)(1-\cos \delta) \leq d^{2}(p, \mu \nu) / 2 T$ and the result follows.

Definition 2.5. Define $\alpha: H \times \bar{H} \times H \rightarrow R$ as follows:

(1) If $x \in H(\infty)$ then $\alpha(p, x, q)=B(V(p, x), q)$ where $V$ is the vector function of Proposition 1.2.

(2) If $x \in H$ then $a(p, x, q)=d(q, x)-d(p, x)$.

This function is a generalization of the $a$-function of Busemann [5, p. 131] and measures the relative distances of finite points $p, q$ to a point $x \in H(\infty)$.

Proposition 2.6. a is a continuous function with respect to the product topology on $H \times \bar{H} \times H$.

Proof. We shall show that for any point $(p, x, q) \in H \times \bar{H} \times H$ and any sequence $\left(p_{n}, x_{n}, q_{n}\right)$ converging to $(p, x, q)$ it follows that $\alpha\left(p_{n}, x_{n}, q_{n}\right)$ converges to $a(p, x, q)$. If suffices to consider only the cases where $x_{n} \subseteq H(\infty)$ for every $n$ and where $x_{n} \subseteq H$ for every $n$ and $d\left(p, x_{n}\right)$ is unbounded.

If $x_{n} \subseteq H(\infty)$ for every $n$, then $\alpha\left(p_{n}, x_{n}, q_{n}\right)=B\left(V\left(p_{n}, x_{n}\right), q_{n}\right) \rightarrow$ $B(V(p, x), q)=\alpha(p, x, q)$ by the continuity of $B$ and $V$. Now suppose that $x_{n} \subseteq H$ for every $n$ and let $v_{n}=V\left(p, x_{n}\right), v=V(p, x), s_{n}=d\left(p, x_{n}\right) . s_{n} \rightarrow+\infty$ and $v_{n}$ is well defined for $n$ sufficiently large. $a\left(p_{n}, x_{n}, q_{n}\right)=d\left(q_{n}, x_{n}\right)-$ $d\left(p_{n}, x_{n}\right)=d\left(q_{n}, \gamma_{v_{n}} s_{n}\right)-s_{n}+d\left(p, x_{n}\right)-d\left(p_{n}, x_{n}\right)=B_{s_{n}}\left(v_{n}, q_{n}\right)+d\left(p, x_{n}\right)-$ $d\left(p_{n}, x_{n}\right) \rightarrow B(v, q)^{n}=B(V(p, x), q)=\alpha(p, x, q)$ since $v_{n} \rightarrow v, p_{n} \rightarrow p, q_{n} \rightarrow q$ and $B_{s_{n}} \rightarrow B$ uniformly on compact subsets by Lemma 2.4 .

Definition 2.7. If $\gamma=\gamma_{v}$ is a geodesic in $H$ then the Busemann function $f_{\gamma}: H \rightarrow R$ is given by $f_{\gamma}(q)=B(v, q)=\lim _{t \rightarrow+\infty} d(q, \gamma t)-t$. 
If $v=V(p, x)$ then $f_{\gamma}(q)=\alpha(p, x, q)$. Busemann functions have the following properties.

Proposition 2.8. (1) For each geodesic $\gamma, f_{\gamma}$ is a uniformly continuous convex function sucb that $\left|f_{\gamma}(p)-f_{\gamma}(q)\right| \leq d(p, q)$.

(2) If $\gamma$ and $\beta$ are asymptotic geodesics then $f_{\gamma}-f_{\beta} \equiv f_{\gamma}(\beta 0)$ in $H$.

(3) If $\gamma$ and $\beta$ are asymptotic geodesics, then $f_{\gamma}(\beta t)-f_{\gamma}(\beta s)=s-t$ for every $s \in R, t \in R$.

Proof. (1) is merely a restatement of Proposition 2.3. To prove (2), let $p$ and $q$ be points in $H$ and $x$ a point in $H(\infty)$ such that $\gamma=\gamma_{p x}$ and $\beta=\gamma_{q x}$. We must show that for any point $r$ in $H, \alpha(p, x, r)-\alpha(q, x, r)=\alpha(p, x, q)$. If $x_{n}$ is any sequence in $H$ converging to $x$, then by Proposition 2.6, $\alpha(p, x, r)=$ $\lim _{n \rightarrow \infty}\left\{d\left(r, x_{n}\right)-d\left(p, x_{n}\right)\right\}$ and $\alpha(q, x, r)=\lim _{n \rightarrow \infty}\left\{d\left(r, x_{n}\right)-d\left(q, x_{n}\right)\right\}$. Hence $\alpha(p, x, r)-\alpha(q, x, r)=\lim _{n \rightarrow \infty}\left\{d\left(q, x_{n}\right)-d\left(p, x_{n}\right)\right\}=\alpha(p, x, q)$ by Proposition 2.6. (3) For any geodesic $\beta, f_{\beta}(\beta t)=-t$. If $\gamma$ and $\beta$ are asymptotic geodesics, $f_{\gamma}(\beta t)-f_{\gamma}(\beta s)=f_{\beta}(\beta t)-f_{\beta}(\beta s)=s-t$ by $(2)$.

If $x \in H(\infty)$ and if $\gamma$ is a geodesic representing $x$, we call $f_{\gamma}$ a Busemann function at $x$. If $f$ is a Busemann function at $x$ and if $p$ is any point in $H$, then the limit sphere at $x$ through $p$ is the set $L(p, x)=\{q \in H: f q=f p\}$. The limit ball at $x$ determined by $p$ is the set $N(p, x)=\{q \in H: f q<f p\}$. By the preceding proposition, these definitions are independent of the choice of the function $f$ at $x$; the Busemann function at $x$ is unique up to an additive constant. It is easy to verify that $L(p, x)$ is the topological boundary of $N(p, x)$ and that $N(p, x)$ is the convex open set $\bigcup_{t>0} N_{t}\left(a_{t}\right)$ where $a=\gamma_{p x}$ and $N_{t}\left(a_{t}\right)$ is the open ball with center $a t$ and radius $t$.

The result below follows easily from the definition of limit sphere and Proposition 2.6.

Proposition 2.9. Let $p, q$ be points in $H, x$ a point in $H(\infty)$.

(1) $q \in L(p, x)$ if and only if $p \in L(q, x)$.

(2) $q \in L(p, x)$ if and only if $p$ and $q$ are equidistant from $x$; that is, if $a_{n}$ is any sequence in $H$ converging to $x$, then $\left|d\left(p, a_{n}\right)-d\left(q, a_{n}\right)\right| \rightarrow 0$.

(3) If $\phi$ is an isometry of $H$ then $\phi L(p, x)=L(\phi p, \phi x)$.

The following observations show that a Busemann function $f$ is indeed a radius function whose value at a point $p$ is the signed distance to the limit sphere $f^{-1}(0)$. (1) and (2) follow from the discussion above and Proposition 2.8. (3) and (4) are Propositions 3.2 and 3.3 of [8].

Let $f$ be a Busemann function at a point $x \in H(\infty)$, and let $L$ be a limit sphere at $x$. Then 
(1) The limit spheres at $x$ are the level sets of $f$.

(2) Each geodesic $\gamma$ representing $x$ meets $L$ precisely once.

(3) Let $\eta_{L}: H \rightarrow L$ be the function that sends $p \in H$ to the unique point at which $\gamma_{p x}$ meets $L$. Then for any $p \in H, \eta_{L}(p)$ is the unique point of $L$ nearest $p$.

(4) Limit spheres at $x$ are parallel; that is, if $L^{\prime}$ is another limit sphere at $x$ and if $p \in L, p^{\prime} \in L^{\prime}$ are arbitrary, then $d\left(L, L^{\prime}\right)=d\left(p, L^{\prime}\right)=d\left(p^{\prime}, L\right)=\mid f L-$ $f L^{\prime}|=| f p-f p^{\prime} \mid$.

Next we show that a limit sphere $L(p, x)$ depends continuously on the points $p$ and $x$.

Proposition 2.10. Let $L(p, x)$ be a limit spbere in $H$, and let $q \in L(p, x)$. For any open set $O$ of $H$ containing $q$ we can find open sets $A$ and $B$ of $H$ and $H(\infty)$ such that $(p, x) \in A \times B$ and if $\left(p^{\prime}, x^{\prime}\right) \in A \times B$ then $L\left(p^{\prime}, x^{\prime}\right) \cap O \neq \varnothing$.

Proof. $\alpha(p, x, q)=0$ where $\alpha$ is the function of Definition 2.5. Given an open set $O$ of $H$ containing $q$, choose $\epsilon>0$ so that $O$ contains the open ball of radius $\epsilon$ and center $q$. By the continuity of $\alpha$ we may choose open sets $A$ and $B$ of $H$ and $H(\infty)$ such that $(p, x) \in A \times B$ and if $\left(p^{\prime}, x^{\prime}\right) \in A \times B$ then $\left|\alpha\left(p^{\prime}, x^{\prime}, q\right)\right|<\epsilon$. These are the desired open sets. Given $\left(p^{\prime}, x^{\prime}\right) \in A \times B$, let $\sigma=\gamma_{q x^{\prime}} . \alpha\left(p^{\prime}, x^{\prime}, \sigma t\right)=-t+\alpha\left(p^{\prime}, x^{\prime}, q\right)$ by Proposition 2.8. If $t_{0}=\alpha\left(p^{\prime}, x^{\prime}, q\right)$ then $\left|t_{0}\right|<\epsilon$ and $\alpha\left(p^{\prime}, x^{\prime}, \sigma t_{0}\right)=0$. Since $\sigma t \in O$ for $|t|<\epsilon$, it follows that $\sigma t_{0} \in L\left(p^{\prime}, x^{\prime}\right) \cap O$.

The Euclidean and hyperbolic spaces represent the two extremes with respect to the geometric properties of their limit spheres. In Euclidean space a limit sphere $L(p, x)$ is a hyperplane through $p$ orthogonal to the direction $x$, and the corresponding limit ball is the open half-space composed of all geodesic rays making an angle $<\pi / 2$ with $\gamma_{p x}$. In hyperbolic space $P$ (disk model), a limit sphere at $x$ is a Euclidean sphere in $P$ that is tangent to the unit sphere $P(\infty)$ at $x$; the only geodesic in the limit ball is the geodesic $\gamma_{p x}$.

We now define a first countable, $T^{1}$ borocycle topology based on limit spheres for $\bar{H}=H \cup H(\infty)$ where $H$ is any Hadamard manifold. If $N$ is a limit ball at $x \in H(\infty)$, we call $N^{x}=N \cup\{x\}$ an augmented limit ball at $x$. The following result is Proposition 3.7 of [8].

Proposition 2.11. There exists a unique (borocycle) topology $b$ on $\bar{H}$ such that

(1) $b$ induces the original topology of $H$, and $H$ is a dense open subset of $\bar{H}$,

(2) for each $x \in H(\infty)$ the set of augmented limit balls at $x$ is a local basis for $b$ at $x$. 
If $D$ is a group of isometries of $H$, we may define a borocyclic limit set $L_{b}(D)$; if $p$ is any point of $H$, then $L_{b}(D)$ is the set of accumulation points in $H(\infty)$ of an orbit $D(p)$ with respect to the horocycle topology $b . L_{b}(D)$ is independent of the point $p$ and invariant under $D . O_{b}(D)=H(\infty)-L_{b}(D)$ is the set of borocyclic ordinary points.

$L_{b}(D)$ need not be closed in $H(\infty)$ with respect to the cone topology and may be empty for even quite complicated groups $D$. If $H$ satisfies Axiom 1 , then for any $x \in H(\infty)$ and any cone neighborhood $W$ of $x$ there exists an augmented limit ball $N^{x}$ such that $N^{x} \subseteq W$. From this it follows that $L_{b}(D) \subseteq L(D)$ (see Propositions 4.8 and 4.9 of [8]). The result below will be useful later.

Proposition 2.12. Let $p_{n}$ be a sequence in $H$ converging to $x \in H(\infty)$ in the borocycle topology. For any point $p \in H, d\left(p_{n}, L(p, x)\right) \rightarrow \infty$.

Proof. Let $p \in H$ and let $L=L(p, x)$. For any number $c>0$ consider the limit ball $N=N\left(\alpha_{c}, x\right)$ where $\alpha=\gamma_{p x}$. Since $p_{n} \rightarrow x$, in the horocycle topology $p_{n} \in N$ for $n$ sufficiently large. If $f$ is the Busemann function $f_{a}$ at $x$, then by remark (4) following Proposition 2.9, $d\left(p_{n}, L\right)=\left|f p_{n}-f p\right| \geq\left|f p_{n}\right|-|f p| \geq c-|f p|$ for $n$ sufficiently large. The result follows since $c>0$ was arbitrary.

3. The sets $W^{s}, W^{s s}, W^{u}$ and $W^{u u}$. Let $M=H / D$ be any complete manifold with curvature $K \leq 0$. For each vector $v \in S M$ we define stable and unstable sets $W^{s}(v), W^{s s}(v), W^{u}(v)$ and $W^{u u}(v)$. These sets generalize the concept of stable and unstable manifolds in modern global analysis and coincide in certain situations, for example if $M$ is compact with $K<0$ [1, p. 30]. The notation is intended to suggest this relationship. The stable and unstable sets are useful tools for the study of the geodesic flow on SM even though it is not clear that the sets are smooth manifolds.

In this section we define the sets and develop some basic properties of them.

Unit vectors $v, w \in S H$ are weakly asymptotic if $\gamma_{v}$ and $\gamma_{w}$ are asymptotic geodesics. $v, w$ are strongly asymptotic if they are weakly asymptotic and if $\mu(v)$ and $\mu(w)$ lie on the same limit sphere determined by $x=\gamma_{v}(\infty)=\gamma_{w}(\infty)$. $v, w$ are weakly (strongly) unstably asymptotic if $-v$ and $-w$ are weakly (strongly) asymptotic. Passing to a quotient manifold $M=H / D$, unit vectors $v, w \in S M$ are weakly asymptotic if they have lifts $v^{*}, w^{*} \epsilon S H$ that are weakly asymptotic. Similarly one defines strong asymptotes and weak (strong) unstable asymptotes in $S M$. For a vector $v \in S M$ (or $S H$ ) let $W^{s}(v), W^{s s}(v), W^{u}(v), W^{u u}(v)$ denote respectively the weak, strong, weak unstable and strong unstable asymptotes of $v$. These sets will be referred to as the stable, strong stable, unstable and strong unstable sets determined by $v$. 
If $\phi$ is an isometry of $H$ it follows from Proposition 2.9(3) and the definition of asymptotes that $\phi_{*} W^{s}(v)=W^{s}\left(\phi_{*} v\right)$ and $\phi_{*} W^{s s}(v)=W^{s s}\left(\phi_{*} v\right)$ for any $v \in S H$. It follows that the stable and unstable sets in $S M$ are projections of those sets in $S H$; that is, $\pi_{*} W^{S}(v)=W^{S}\left(\pi_{*} v\right)$ for any $v \in S H$, and similar statements are true for the other three sets. All four asymptote relations are equivalence relations on the vectors in $S M$ and $S H$.

Proposition 3.1. Let $M=H / D$ be a complete manifold with $K \leq 0$. Let $T_{t}$ denote the geodesic flow in both $S M$ and $S H$, and let $v \in S M$ or $S H$ be arbitrary. If $A$ is any set in $S M$ or $S H$ let $\bar{A}$ denote the closure of $A$ in $S M$ or $S H$.

(1) For any $t \in R, T_{t} W^{s}(v)=W^{s}(v)=W^{s}\left(T_{t} v\right)$ and $T_{t} W^{s s}(v)=W^{s s}\left(T_{t} v\right)$.

(2) If $w \in W^{s}(v)$, then $T_{t} w \in W^{s s}(v)$ for some $t \in R$.

(3) If $v \in S M$ is periodic with period $\omega>0$ and if $w \in \overline{W^{S}(v)}$ then for some number $0 \leq c \leq \omega, T_{c} w \in \overline{W^{s s}(v)}$.

(4) If $w \in W^{s s}(v)$ (respectively $w \in W^{s}(v)$ ) then $W^{s s}(v)=W^{s s}(w)$ (respectively $\left.W^{s}(v)=W^{s}(w)\right)$.

(5) $W^{u}(-v)=-W^{s}(v)$ and $W^{u u}(-v)=-W^{s s}(v)$.

(6) (Continuity of the sets). Let $w \in W^{s s}(v)$ (respectively $w \in W^{s}(v)$ ) and let $O$ be an open set (of $S M$ or $S H$ ) containing $w$. Then there exists an open set $U$ containing $v$ sucb that if $v^{\prime} \in U$ then $W^{s s}\left(v^{\prime}\right) \cap O \neq \varnothing$ (respectively $\left.W^{s}\left(v^{\prime}\right) \cap O \neq \varnothing\right)$.

(7) If $w \in \overline{W^{s s}(v)}$, then $\overline{W^{s s}(w)} \subseteq \overline{W^{s s}(v)}$. If $w \in \overline{W^{s}(v)}$, then $\overline{W^{s}(w)} \subseteq \overline{W^{s}(v)}$.

Remark 3.2. (1) Analogous results are true for the sets $W^{u}$ and $W^{u u}$ in (1), (2), (4), (6) and (7). The proofs follow from the relations in (5) and the statements for $W^{s}$ and $W^{s s}$.

(2) In parts (1), (2), (4) and (6) of the proposition it suffices to consider the case where $v \in S H$. One reduces to this case by keeping in mind the following facts: (a) $\pi_{*}: S H \rightarrow S M$ is a surjective local homeomorphism and carries open sets in $S H$ onto open sets in $S M$. (b) If $T_{t}$ again denotes the geodesic flow in both $S H$ and $S M$ then $\pi_{*} \circ T_{t}=T_{t} \circ \pi_{*}$. (c) For any $v \in S H$, $\pi_{*} W^{s}(\nu)=W^{s}\left(\pi_{*} v\right)$ and similarly the sets $W^{s s}, W^{u}$ and $W^{u u}$ in $S M$ are projections of the corresponding sets in $\mathrm{SH}$.

Proof of Proposition 3.1. We assume in (1), (2), (4) and (6) that $v \in S H$.

(1) It suffices to prove that, for any $t \in R, T_{t} W^{s}(\nu) \subseteq W^{s}(v)=W^{s}\left(T_{t} v\right)$ and $T_{t} W^{s s}(v) \subseteq W^{s s}\left(T_{t} v\right)$. If $w \in T_{t} W^{s}(v)$ then $T_{-t} w \in W^{s}(v)$ and the geodesics $\gamma_{T-t} w$ and $\gamma_{\nu}$ are asymptotic. Therefore $\gamma_{\nu}$ and $\gamma_{w}$ are asymptotic and $w \epsilon$ $W^{s}(v)$. The same argument shows that $W^{s}(\nu)=W^{s}\left(T_{t} v\right)$.

Now let $w \in W^{s s}(\nu)$; let $x=\gamma_{v}(\infty)=\gamma_{w}(\infty)$ and let $f$ be a Busemann function at $x$. By assumption $f\left(\gamma_{v} 0\right)=f\left(\gamma_{w} 0\right)$ and, by Proposition 2.8, $f\left(\gamma_{w} t\right)=$ 
$-t+f\left(\gamma_{w} 0\right)=-t+f\left(\gamma_{v} 0\right)=f\left(\gamma_{v} t\right)$. Since the points $\mu\left(T_{t} w\right)$ and $\mu\left(T_{t} v\right)$ lie on the same limit sphere determined by $x$ and since $T_{t} w \in W^{s}(v)=W^{s}\left(T_{t} v\right)$ we see that $T_{t} w \in W^{s s}\left(T_{t} v\right)$.

(2) Let $w \in W^{s}(v), x=\gamma_{\nu}(\infty)$ and $f$ be a Busemann function at $x$. Let $t=$ $f\left(\gamma_{w} 0\right)-f\left(\gamma_{v} 0\right)$. Then $f\left(\gamma_{w} t\right)=-t+f\left(\gamma_{w} 0\right)=f\left(\gamma_{v} 0\right)$. The points $\mu\left(T_{t} w\right)$ and $\mu(v)$ lie on the same limit sphere determined by $x$ and since $T_{t} w \in W^{s}(\nu)$ it follows by definition that $T_{t} w \in W^{s s}(v)$.

(3) Let $v$ be periodic with period $\omega>0$ and let $w \in \overline{W^{s}(v)}$. Let $v_{n}$ be a sequence in $W^{s}(v)$ such that $v_{n} \rightarrow w$. For each $n$ there exists a number $t_{n}$ such that $T_{t_{n}} v_{n} \in W^{S S}(\nu)$ by (2). Choose integers $a_{n}$ such that $a_{n} \omega \leq t_{n}<\left(a_{n}+1\right)_{\omega}$ and let $c_{n}=t_{n}-a_{n} \omega$. Then $T_{c_{n}{ }_{n}}=T_{t_{n}-a_{n} \omega_{n}} \in W^{s s}\left(T_{-a_{n}} \omega^{\nu}\right)=W^{s s}(\nu)$ by (1). Passing to a subsequence let $c_{n} \rightarrow c$. Then $T_{c_{n}} v_{n} \rightarrow T_{c} w \in \overline{W^{s s}(v)}$.

(4) This follows immediately from the fact that the asymptote relations are equivalence relations in $S H$ and $S M$.

(5) This is a restatement of the definitions of the sets $W^{u}$ and $W^{u u}$.

(6) Let $v \in S H, w \in W^{S S}(\nu)$ and let $O$ be an open set of $S H$ containing $w$. If $x=\gamma_{v}(\infty)$ let $p$ and $q$ be points in $H$ such that $w=V(q, x), v=V(p, x)$ and $q \in L(p, x)$. By the continuity of $V$ (Proposition 1.2), we may choose open sets $A$ and $B$ of $H$ and $H(\infty)$ such that $(q, x) \in A \times B$ and $V(A \times B) \subseteq O$. By Proposition 2.10 we may choose open sets $C$ and $D$ of $H$ and $H(\infty)$ such that $(p, x)$ $\epsilon C \times D$ and if $\left(p^{\prime}, x^{\prime}\right) \in C \times D$, then $L\left(p^{\prime}, x^{\prime}\right) \cap A \neq \varnothing$. If $U=V(C \times(B \cap D))$, then $U$ is an open set in $S H$ containing $v$ since $V: H \times H(\infty) \rightarrow S H$ is a homeomorphism. If $v^{\prime} \in U$, then $v^{\prime}=V\left(p^{\prime}, x^{\prime}\right)$ for some $\left(p^{\prime}, x^{\prime}\right) \in C \times(B \cap D)$. By the choice of $C$ and $D$ there exists $q^{\prime} \in L\left(p^{\prime}, x^{\prime}\right) \cap A$, and by the choice of $A$ and $B, V\left(q^{\prime}, x^{\prime}\right) \in W^{s s}\left(v^{\prime}\right) \cap O$.

Now let $w \in W^{S}(v)$ and let $O$ be an open set of $S H$ containing $w$. Choose $t \in R$ such that $T_{t} w \in W^{s s}(v)$, and let $U$ be an open set of $S H$ containing $v$ such that if $v^{\prime} \in U$ then $W^{s s}\left(v^{\prime}\right) \cap T_{t} O \neq \varnothing$. If $v^{\prime} \in U$, then $W^{s}\left(v^{\prime}\right) \cap O \supseteq$ $T_{-t} W^{s s}\left(v^{\prime}\right) \cap O \neq \varnothing$.

(7) Let $v \in S M$ (or $S H$ ) and let $w \in \overline{W^{S S}(v)}$. Let $v^{*} \in \overline{W^{S S}(w)}$ and let $O$ be an open set (of $S M$ or $S H$ ) containing $v^{*}$. Since $W^{s s}(w) \cap O \neq \varnothing$, we may choose an open set $U$ containing $w$ such that, for every $v^{\prime} \in U, W^{s s}\left(v^{\prime}\right) \cap O \neq \varnothing$. Since $w \in \widehat{W^{s s}(v)}$ there exists $v^{\prime} \in W^{s s}(v) \cap U$, and therefore $W^{s s}(v) \cap 0=$ $W^{s s}\left(v^{\prime}\right) \cap O \neq \varnothing$. Since $O$ was arbitrary, $v^{*} \in \overline{W^{s s}(v)}$, and since $v^{*}$ was arbi-

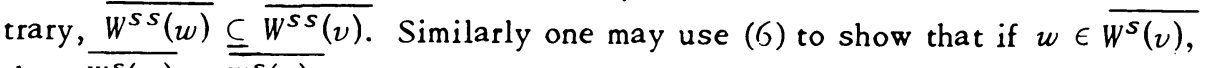
then $\overline{W^{s}(w)} \subseteq \overline{W^{s}(v)}$

4. The relationship of the sets $W^{s}, W^{s s}, W^{u}$ and $w^{u u}$ to the geodesic flow on $S M$. We first list some basic properties and dynamical concepts for the geodesic flow $T_{t}$ on $S M, M=H / D$, and relate these to the stable and unstable 
sets. Propositions 4.6 and 4.11 exhibit fundamental relations.

In Theorems 4.14 and 4.16 we formulate the problems of topological transitivity and topological mixing in terms of the stable and unstable sets. It seems very likely that ergodicity and metrical mixing can also be formulated in terms of these sets, but this has not been investigated. Theorem 4.16 is a corollary of Theorem 4.15, in which we do not assume that every point of $S M$ is nonwandering.

The method used to investigate topological mixing is a generalization of that used by Hedlund for surfaces ([11], [12]). The discussion on pp. 248-250 of [10] is a good geometric description of the importance of the sets $W^{s s}$. Proposition 4.5 contains the basic idea of this discussion. The existence of a vector $v \in S M$ such that $W^{S S}(v)$ is dense in $S M$ is equivalent to the existence of a transitive horocycle in Hedlund's terminology.

Definition 4.1. If $v \in S M, P^{+}(v)=\{w \in S M$ : for any neighborhoods $O, U$ in $S M$ of $v, w$ respectively we can find a sequence $t_{n} \subseteq R, t_{n} \rightarrow+\infty$ such that $T_{t_{n}}(O) \cap U \neq \varnothing$ for every $\left.n\right\} . \quad P^{+}(v)$ is the positive prolongational limit set of $v[2]$.

Similarly one defines $P^{-}(v)$, the negative prolongational limit set of $v$, by requiring in the definition above that $t_{n} \rightarrow-\infty . w \in P^{+}(v)$ if and only if $v \in$ $P^{-}(w)$. Equivalently one may define $P^{+}(v)$ (respectively $P^{-}(v)$ ) to be $\{w \in S M$ : there exist sequences $t_{n} \subseteq R$ and $v_{n} \subseteq S M$ such that $t_{n} \rightarrow+\infty$ (respectively $\left.t_{n} \rightarrow-\infty\right), v_{n} \rightarrow v$ and $\left.T_{t_{n}} v_{n} \rightarrow w\right\}$.

Definition 4.2. $v \in S M$ is nonwandering if $v \in P^{+}(v)$ (equivalently $v \in P^{-}(v)$ ). Let $\Omega$ denote the set of nonwandering points in $S M$.

For any $v \in S M, P^{+}(v), P^{-}(v)$ and $\Omega$ are closed subsets of $S M$ invariant under $T_{t}$. It is easy to verify the following properties of the geodesic flow. If $t \in R$ and $v \in S M$ are arbitrary then

(1) $T_{t}(-v)=-T_{-t} v$,

(2) $P^{-}(-v)=-P^{+}(v), P^{+}(-v)=-P^{-}(v)$,

(3) $\Omega=-\Omega$.

The following idea will be convenient in the discussion of topological mixing.

Definition 4.3. Let $t_{n} \subseteq R$ be a sequence such that $t_{n} \rightarrow+\infty$ or $t_{n} \rightarrow-\infty$. For vectors $v, w \in S M$ we say that $v$ is $t_{n}$ related to $w$ if for any neighborhoods $O, U$ in $S M$ of $v, w$ respectively, $T_{t_{n}}(O) \cap U \neq \varnothing$ for $n$ sufficiently large.

Using the definitions and the facts following Definition 4.2 we easily obtain

Proposition 4.4. Let $M=H / D$ be a complete manifold with $K \leq 0$.

(1) If $v, w \in S M$, then $w \in P^{+}(v)$ if and only if $v$ is $t_{n}$ related to $w$ for some sequence $t_{n} \subseteq R, t_{n} \rightarrow+\infty$.

(2) $v$ is $t_{n}$ related to $w$ if and only if $w$ is $-t_{n}$ related to $v$.

(3) $v$ is $t_{n}$ related to $w$ if and only if $-w$ is $t_{n}$ related to $-v$. 
(4) For each $v \in S M$ and each sequence $t_{n} \subseteq R$ sucb that $t_{n} \rightarrow+\infty$ or $t_{n} \rightarrow-\infty\left\{w \in S M: v\right.$ is $t_{n}$ related to $\left.w\right\}$ is closed in SM.

(5) For each $w \in S M$ and each sequence $t_{n} \subseteq R$ such that $t_{n} \rightarrow+\infty$ or $t_{n} \rightarrow-\infty\left\{v \in S M: v\right.$ is $t_{n}$ related to $\left.w\right\}$ is closed in SM.

(6) $v$ is $t_{n}$ related to $w$ if and only if for each subsequence $s_{n}$ of $t_{n}$ there exists a further subsequence $r_{n}$ of $s_{n}$ and a sequence $v_{n} \subseteq S M$ such that $v_{n} \rightarrow$ $v$ and $T_{r_{n}} v_{n} \rightarrow w$.

Proposition 4.5. Let $M=H / D$, and let $v \in S M$ be $t_{n}$ related to $w \in S M$ for some sequence $\underline{t_{n} \subseteq R,} t_{n} \rightarrow+\infty$.

(1) If $v^{*} \in \underline{W^{s s}(v)}$ then $v^{*}$ is $t_{n}$ related to $w$.

(2) If $w^{*} \in \overline{W^{u u}(w)}$ then $v$ is $t_{n}$ related to $w^{*}$.

(3) If $v^{*} \in \overline{W^{s s}(v)}$ and $w^{*} \in \overline{W^{u u}(w)}$ then $v^{*}$ is $t_{n}$ related to $w^{*}$.

Proof. (1) By (5) of the preceding result we need only show that, for any $v^{*} \in W^{s s}(v), v^{*}$ is $t_{n}$ related to $w$. By (6) of the preceding result it suffices to show that for any subsequence $s_{n}$ of $t_{n}$ we can find a subsequence $r_{n}$ of $s_{n}$ and a sequence $v_{n}^{*} \subseteq S M$ such that $v_{n}^{*} \rightarrow v^{*}$ and $T_{r_{n}} v_{n}^{*} \rightarrow w$.

Let $v^{*} \in W^{s s}(v)$ and let a subsequence $s_{n}$ of $t_{n}$ be given. Since $v$ is $t_{n}$ related to $w$ we can find a subsequence $r_{n}$ of $s_{n}$ and a sequence $v_{n} \subseteq S M$ such that $v_{n} \rightarrow v$ and $T_{r_{n}} v_{n} \rightarrow w$. The sequence $r_{n}$ will also work for $v^{*}$. Let $V(p, x), V(q, x)$ and $V\left(q_{n}, x_{n}\right) \in S H$ be lifts of $v^{*}, v$ and $v_{n}$ respectively such that $V\left(q_{n}, x_{n}\right) \rightarrow V(q, x)$ and $p \in L(q, x) . q_{n} \rightarrow q$ and $x_{n} \rightarrow x$ by Proposition 1.2. If $\alpha=\gamma_{V(q, x)}$ and $\alpha_{n}=\gamma_{V\left(q_{n}, x_{n}\right)}$, then $\alpha_{n}^{\prime}(0) \rightarrow \alpha^{\prime}(0)$ and, by Proposition 1.4, $a_{n}\left(r_{n}\right) \rightarrow a(\infty)=x$.

If $a_{n}=d\left(p, \alpha_{n} r_{n}\right)$ we show that $\left|a_{n}-r_{n}\right| \rightarrow 0$. Since $r_{n}=d\left(q_{n}, a_{n} r_{n}\right)$ and $q_{n} \rightarrow q$ we see that $\left|d\left(q, \alpha_{n} r_{n}\right)-r_{n}\right| \rightarrow 0$. By Proposition 2.9, $\left|a_{n}-d\left(q, a_{n} r_{n}\right)\right|$ $=\left|d\left(p, a_{n} r_{n}\right)-d\left(q, a_{n} r_{n}\right)\right| \rightarrow 0$ since $p \in L(q, x)$ and $a_{n} r_{n} \rightarrow x$. Thus $\left|a_{n}-r_{n}\right| \rightarrow 0$.

The vectors $T_{a_{n}} V\left(p, \alpha_{n} r_{n}\right)$ and $T_{r_{n}} V\left(q_{n}, x_{n}\right)$ are tangent to $H$ at $\alpha_{n} r_{n}$, and the angle that they subtend $\rightarrow 0$ as $n \rightarrow \infty$ by the remark following Proposition 2.1 since $d\left(p, q_{n}\right)$ is bounded. If $v_{n}^{*}=\pi_{*} V\left(p, a_{n}{ }_{n}\right)$, then $\lim _{n \rightarrow \infty} T_{a_{n}} v_{n}^{*}=$ $\lim _{n \rightarrow \infty} T_{r_{n}} v_{n}=w$. Furthermore, $v_{n}^{*} \rightarrow v^{*}=\pi_{*} V(p, x)$ since $\alpha_{n}{ }^{r}{ }_{n} \rightarrow x$. Finally $\lim _{n \rightarrow \infty} T_{r_{n}}^{n} v_{n}^{*}=\lim _{n \rightarrow \infty} T_{a_{n}} v_{n}^{*}=w$. This proves that $v^{*}$ is $t_{n}$ related to $w$.

(2) If $w^{*} \in \overline{W^{u u}(w)}$ then $-w^{*} \epsilon-\overline{W^{u u}(w)}=\overline{W^{s s}(-w)}$. Since $v$ is $t_{n}$ related to $w,-w$ is $t_{n}$ related to $-v$ by (3) of the preceding result. Therefore $-w^{*}$ is $t_{n}$ related to $-v$ by (1) and $v$ is $t_{n}$ related to $w^{*}$.

(3) This follows immediately from (1) and (2).

Proposition 4.6. Let $M=H / D$ and let $v, w \in S M$.

(1) If $w \in P^{+}(v)$ then $\overline{W^{u}(w)} \subseteq P^{+}(v)$.

(2) If $w \in P^{-}(v)$ then $\overline{W^{s}(w)} \subseteq P^{-}(v)$. 
Proof. We shall prove only (2) since the proof of (1) is similar. Let $w \epsilon$ $P^{-}(v)$ and let $w^{*} \in W^{s}(w)$. For some sequence $t_{n} \subseteq R$ such that $t_{n} \rightarrow+\infty, w$ is $t_{n}$ related to $v$ since $v \in P^{+}(w)$. For some $t \in R, T_{t} w^{*} \in W^{s s}(w)$ and $T_{t} w^{*}$ is $t_{n}$ related to $v$ by the preceding result. Therefore, $w^{*}$ is $\left(t_{n}+t\right)$ related to $v$ and $v \in P^{+}\left(w^{*}\right)$ or $w^{*} \in P^{-}(v)$. $\overline{W^{s}(w)} \subseteq P^{-}(v)$ since $w^{*}$ was arbitrary and $P^{-}(v)$ is closed in $S M$.

In Proposition 4.11 we shall clarify further the relationship between $P^{+}, P^{-}$ and the stable and unstable sets. For this purpose it is useful to characterize the sets $P^{+}$and $P^{-}$in terms of the action of $D$ on $H(\infty)$. The following idea is discussed in [7] and [8] and details may be found there.

Definition 4.7. Let $D$ be a properly discontinuous group of isometries of $H$. Points $x, y \in H(\infty)$, not necessarily distinct, are dual relative to $D$ if there exists a sequence $\phi_{n} \subseteq D$ such that $\phi_{n} p \rightarrow x$ and $\phi_{n}^{-1} p \rightarrow y$ for any point $p \in H$.

Note that for any point $q \in H, d\left(\phi_{n} p, \phi_{n} q\right)=d(p, q)$ for every $n$, and hence, for any point $r \in H, \chi_{r}\left(\phi_{n} p, \phi_{n} q\right) \rightarrow 0$ by the observation following Proposition 2.1. Hence $\phi_{n} q \rightarrow x$ for any point $q \in H$.

Dual points must lie in $L(D)$ and any point in $L(D)$ has at least one dual point. It is shown in [7] that the set of points dual to a given point $x$ is closed in $H(\infty)$ and invariant under $D$. The results below are Propositions 2.6, 3.7 and 3.8 of [7]. See also Proposition 8.7 of [8].

Proposition 4.8. Let $H$ satisfy Axiom 1 and let $D$ be a group of isometries of $H$ such that $L(D)$ bas more than two points. Then any two points of $L(D)$, not necessarily distinct, are dual.

Proposition 4.9. Let $M=H / D$ be an arbitrary complete manifold with $K \leq$ 0 . Let $v^{*}, w^{*} \in S H$ be arbitrary lifts of vectors $v, w \in S M$. Then

(1) $w \in P^{+}(v)$ if and only if $\gamma_{v^{*}}(\infty)$ and $\gamma_{w^{*}}(-\infty)$ are dual relative to $D$.

(2) $w \in P^{-}(v)$ if and only if $\gamma_{v^{*}}(-\infty)$ and $\gamma_{w^{*}}(\infty)$ are dual relative to $D$.

Proposition 4.10. Let $M=H / D$ where $H$ satisfies Axiom 1 and $L(D)$ bas more than two points. Let $v^{*} \in S H$ be an arbitrary lift of a vector $v \in S M$. Then $v \in \Omega$ if and only if $\gamma_{\nu^{*}}(\infty)$ and $\gamma_{\nu^{*}}(-\infty)$ are both in $L(D)$.

We are now ready to complement Proposition 4.6.

Proposition 4.11. Let $M=H / D$ be an arbitrary complete manifold with $K$ $\leq 0$ and let $v \in S M$. Then

(1) $P^{-}(v) \subseteq \overline{W^{s}(v)}$ with equality if and only if $v \in \Omega$.

(2) $P^{+}(\nu) \subseteq \overline{W^{u}(v)}$ with equality if and only if $v \in \Omega$. 
Proof. (1) Let $w \in P^{-}(v)$ and let $v^{*}, w^{*} \in S H$ be lifts of $v$ and $w$. By a preceding result, $\gamma_{v^{*}}(-\infty)$ and $\gamma_{w^{*}}(\infty)$ are dual relative to $D$. Let $p=\gamma_{v^{*}}(0)$, $x=\gamma_{v^{*}}(\infty)$ and choose a sequence $\phi_{n} \subseteq D$ such that $\phi_{n}^{-1} p \rightarrow \gamma_{v^{*}}(-\infty)$ and $\phi_{n} p \rightarrow \gamma_{w^{*}}(\infty)$. We first show that $\phi_{n} x \rightarrow \gamma_{w^{*}}(\infty)$. It is a standard result that the sum of the interior angles of a geodesic triangle in $H$ is $\leq \pi$. This result is also valid if one vertex is in $H(\infty)$ by the discussion preceding Remark 2.1 of [8]. Thus $\chi_{\phi_{n}^{-1}} p_{p}(p, x)+\not_{p}\left(\phi_{n}^{-1} p, x\right) \leq \pi$. Since $\not_{p}\left(\phi_{n}^{-1} p, x\right)+$ $\chi_{p}\left(\phi_{n}^{-1} p, \gamma_{v^{*}}(-\infty)\right)^{n}=\pi$, it follows that $\chi_{\phi_{n}^{-1} p}(p, x) \leq \chi_{p}\left(\phi_{n}^{-1} p, \gamma_{v^{*}}(-\infty)\right) \rightarrow$ 0 by the choice of $\phi_{n}$. Finally $\chi_{p}\left(\phi_{n} x, y_{w^{*}}(\infty)\right) \leq \chi_{p}\left(\phi_{n} x, \phi_{n} p\right)+$ $\chi_{p}\left(\phi_{n} p, \gamma_{w^{*}}(\infty)\right)=\not_{\phi_{n}^{-1} p}(x, p)+\not_{p}\left(\phi_{n} p, \gamma_{w^{*}}(\infty)\right) \rightarrow 0$. Therefore $\phi_{n} x \rightarrow$ $\gamma_{w *}(\infty)$.

Let $q=\gamma_{w^{*}}(0)$ and let $v_{n}=\pi_{*} V\left(q, \phi_{n} x\right)=\pi_{*} \circ\left(\phi_{n}\right)_{*} v\left(\phi_{n}^{-1} q, x\right)=$ $\pi_{*} V\left(\phi_{n}^{-1} q, x\right) . v_{n} \in W^{s}(v)$ for every $n$ and $v_{n} \rightarrow w=\pi_{*} w^{*}=\pi_{*} V\left(q, \gamma_{w^{*}}(\infty)\right)$ since $\phi_{n} x \rightarrow \gamma_{w^{*}}(\infty)$. Hence $w \in \overline{W^{s}(v)}$ and since $w$ was arbitrary in $P^{-}(v)$,

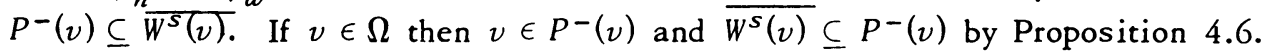
Therefore $P^{-}(v)=\overline{W^{s}(v)}$. Conversely if $P^{-}(v)=\overline{W^{s}(v)}$ then $v \in P^{-}(v)$ since $v \in W^{s}(v)$.

(2) $P^{+}(v)=-P^{-}(-v) \subseteq \overline{-W^{s}(-v)}=\overline{W^{u}(v)}$ by (1), Proposition 3.1 and the facts following Definition 4.2. If $v \in P^{+}(v)$ then $\overline{W^{u}(v)} \subseteq P^{+}(v)$ by Proposition 4.6. If $P^{+}(v)=\overline{W^{u}(v)}$ then $v \in P^{+}(v)$ since $v \in W^{u}(v)$.

We now define topological transitivity and topological mixing for any complete flow and apply the results above to reformulate these problems for the geodesic flow on $S M$.

If $G$ is a group of homeomorphisms of a second countable, locally compact, Hausdorff space $X$ then standard arguments show that the following assertions are equivalent ([6, Proposition 3.4] or [3, pp. 206-207]):

(1) For some $x \in X,\{g(x): g \in G\}$ is dense in $X$.

(2) For any two open sets $O, U$ of $X$, there exists $g \in G$ such that $g(O)$ $\cap U \neq \varnothing$.

Definition 4.12. The action of $G$ on $X$ is topologically transitive if either of the conditions above is satisfied.

If $G=T_{t}$, a complete flow, (one parameter group of homeomorphisms) then we may define a stronger property. Roughly this property says that with respect to both the past and the future an open set $O$ disperses itself homogeneously throughout the space $X$.

Definition 4.13. If $T_{t}$ is a complete flow on $X$ then the action of $T_{t}$ on $X$ is topologically mixing if for any two open sets $O, U$ of $X$ there exists a number $A>0$ such that, for $|t| \geq A, T_{t}(O) \cap U \neq \varnothing$. 
Theore $\mathrm{m}$ 4.14. Let $M=H / D$ be an arbitrary complete manifold with curvature $K \leq 0$ and let $\Omega=S M$. Then the following conditions are equivalent:

(1) The geodesic flow is topologically transitive on SM.

(2) $D$ is topologically transitive on $H(\infty)$.

(3) For every $x \in H(\infty)$ the orbit $D(x)$ is dense in $H(\infty)$.

(4) For some $v \in S M, \overline{W^{S}(v)}=S M$.

(5) For every $v \in S M, \overline{W^{S}(v)}=S M$.

(6) For any two open sets $O, U$ in $S M$ there exists a sequence $t_{n} \subseteq R$, $t_{n} \rightarrow+\infty$ such that $T_{t_{n}}(O) \cap U \neq \varnothing$ for every $n$.

We shall prove the se assertions in the order (1) $\Rightarrow(2) \Rightarrow(4) \Rightarrow(6) \Rightarrow(5) \Rightarrow$ (3) $\Rightarrow(1)$.

$(1) \Longrightarrow(2)$. Let $T_{t}$ denote the geodesic flow on $S M$ and let $v \in S M$ have a $T_{t}$ orbit dense in $S M$. If $V(p, x) \in S H$ is a lift of $v$ we show that the $D$ orbit of $x$ is dense in $H(\infty)$. Given $y \in H(\infty)$ let $w=\pi_{*} V(p, y)$ and choose a sequence $t_{n} \subseteq R$ such that $T_{t_{n}} v \rightarrow w$. If $\gamma=\gamma_{V(p, x)}$ then there exists a sequence $\phi_{n} \subseteq$ $D$ such that $\left(\phi_{n} \circ \gamma\right)\left(t_{n}\right) \rightarrow V(p, y)$. Let $\alpha_{n}$ be the geodesic given by $\alpha_{n}(t)=$ $\left(\phi_{n} \circ \gamma\right)\left(t_{n}+t\right)$. Then $\alpha_{n}^{\prime}(0) \rightarrow V(p, y)$ and $\alpha_{n}(\infty)=\left(\phi_{n} \circ \gamma\right)(\infty)=\phi_{n} x$. Since $\alpha_{n}^{\prime}(0)=V\left(\alpha_{n}(0), \alpha_{n}(\infty)\right)$ it follows by Proposition 1.2 that $\phi_{n} x=\alpha_{n}(\infty) \rightarrow y$. Therefore $y \in \overline{D(x)}$, and we are done since $y$ was arbitrary.

$(2) \Rightarrow(4)$. Let $x \in H(\infty)$ be chosen so that $D(x)$ is dense in $H(\infty)$. Fix a point $p \in H$ and let $v=\pi_{*} V(p, x)$. We show that $W^{S}(v)$ is dense in SM. Let $w \in S M$ have a lift $V(q, y) \in S H$. Choose a sequence $\phi_{n} \subseteq D$ such that $\phi_{n} x \rightarrow y$ and let $w_{n}=\pi_{*} V\left(q, \phi_{n} x\right)=\pi_{*} V\left(\phi_{n}^{-1} q, x\right)$. By the continuity of $V, w_{n} \rightarrow w$ and $w_{n} \in W^{s}(v)$ for every integer $n$.

$(4) \Rightarrow(6)$. Let $v \in S M$ be given such that $\overline{W^{s}(v)}=S M$. Let $v^{\prime}, w^{\prime}$ be any vectors in $S M$ and let $w_{n} \subseteq W^{s}(v)$ be a sequence converging to $w^{\prime}$. Since $W^{s}\left(w_{n}\right)$ $=W^{S}(v)$ and $\Omega=S M$, it follows by Proposition 4.11 that $P^{-}\left(w_{n}\right)=\overline{W^{S}\left(w_{n}\right)}=S M$ for every $n$. $v^{\prime} \in P^{-}\left(w_{n}\right)$ or $w_{n} \in P^{+}\left(v^{\prime}\right)$ for every $n$, and therefore $w^{\prime} \in P^{+}\left(v^{\prime}\right)$ since $w_{n} \rightarrow w^{\prime}$ and $P^{+}\left(v^{\prime}\right)$ is closed. We have shown that $P^{+}\left(v^{\prime}\right)=S M$ for any $\nu^{\prime} \in S M$. This is a restatement of (6).

$(6) \Longrightarrow(5)$. For every $v \in S M, \overline{W^{S}(v)}=P^{-}(v)$ since $\Omega=S M$. Since $P^{+}(v)=$ $-P^{-}(-v)$ and $S M=-S M$, it is clear that (6) and (5) are equivalent statements.

$(5) \Rightarrow(3)$. Let $x, y$ be any two points of $H(\infty)$. Let $p$ be any point in $H$ and let $v=\pi_{*} V(p, x), w=\pi_{*} V(p, y)$. Since $w \in \overline{W^{S}(v)}=S M$ there exists a sequence $v_{n} \subseteq W^{s}(v)$ such that $v_{n} \rightarrow w$. Choose a sequence $\phi_{n} \subseteq D$ and a sequence $p_{n} \subseteq H$ such that $\pi_{*} V\left(p_{n}, \phi_{n} x\right)=v_{n}$ and $V\left(p_{n}, \phi_{n} x\right) \rightarrow V(p, y)$. By Proposition $1.2 \phi_{n} x \rightarrow y$ and the result is proved since $x$ and $y$ were arbitrary.

(3) $\Rightarrow(1)$. In the proof that (6) $\Rightarrow(5)$ we also showed that (5) $\Rightarrow(6)$. (6) $\Rightarrow$ (1) by one of the equivalent definitions of topological transitivity. We need only 
show that (3) $\Rightarrow(5)$, and this follows exactly as in the proof of $(2) \Rightarrow(4)$.

For a manifold $M=H / D$ let $\Omega^{*}=U_{v \in \Omega^{W}}(v)$. Because of Proposition 3.1 (1) and (2) and the fact that $T_{t} \Omega=\Omega$ for every $t \in R$ it is easy to show that $\Omega^{*}$ $=U_{v \in \boldsymbol{\Omega}} W^{s s}(v)$.

Theorem 4.15. Let $M=H / D$ be an arbitrary complete manifold with $K \leq 0$ and let $\Omega \subseteq S M$ contain a periodic vector. Suppose that, for every $v \in \Omega, \Omega \subseteq$ $\overline{W^{s}(v)}$ and that, for some $v \in \Omega, \Omega \subseteq \overline{W^{s s}(v)}$. Then

(1) For any open sets $O^{*}, U^{*}$ of $S M$ such that $O^{*} \cap \Omega^{*} \neq \varnothing$ and $U^{*} \cap \Omega^{*}$ $\neq \varnothing$ there exists $A>0: t \geq A$ implies $T_{t}\left(O^{*}\right) \cap\left(-U^{*}\right) \neq \varnothing$.

(2) For any open sets $O^{*}, U$ of $S M$ such that $O^{*} \cap \Omega^{*} \neq \varnothing$ and $U \cap \Omega \neq$ $\varnothing$ there exists $A>0: t \geq A$ implies $T_{t}\left(O^{*}\right) \cap U \neq \varnothing$.

(3) For any open sets $O, U$ of $S M$ such that $O \cap \Omega \neq \varnothing$ and $U \cap \Omega \neq \varnothing$ there exists $A>0:|t| \geq A$ implies $T_{t}(O) \cap U \neq \varnothing$.

Since $W^{s s}(v) \subseteq W^{s}(v)$ for any $v \in S M$ we obtain as an immediate corollary of this result and the preceding one:

Theorem 4.16. Let $M=H / D$ be an arbitrary complete manifold with $K \leq 0$. Suppose that $\Omega=S M, \Omega$ contains a periodic vector, and for some $v \in S M, \overline{W^{5 S}(\bar{v})}$ =SM. Then the geodesic flow on SM is topologically mixing.

Remark 4.17. If $M$ is compact then it is always true that $\Omega=S M$ and $\Omega$ contains a periodic vector. The condition $\Omega=S M$ may be implied by the condition $\overline{W^{s s}(v)}=S M$.

Before proving Theorem 4.15 we need the following

Lemma 4.18. Let the hypotheses of Theorem 4.15 be satisfied and let $v^{*} \epsilon$ $\Omega$ be periodic. Then $\Omega \subseteq \overline{W^{s s}\left(\nu^{*}\right)}$.

Proof. Choose $v \in \Omega$ such that $\overline{W^{s s}(v)} \supseteq \Omega$. If $\omega>0$ is the period of $v^{*}$ then, for some number $0 \leq c \leq \omega, T_{c} v \in \overline{W^{S S}\left(v^{*}\right)}$ by Proposition 3.1 and the fact that $v \in \Omega \subseteq \overline{W^{s}\left(v^{*}\right)}$. By Proposition 3.1, (7) $\overline{W^{s s}\left(T_{c} v\right)} \subseteq \overline{W^{s s}\left(v^{*}\right)}$. However, $\Omega$ $=T_{c} \Omega \subseteq T_{c} \overline{W^{S S}(\nu)}=\overline{W^{S S}\left(T_{c} v\right)}$.

Proof of 'Theorem 4.15. (1) It suffices to show that for any vectors $v^{*}, w^{*}$ $\epsilon \Omega^{*}$ and, for any sequence $t_{n} \subseteq R, t_{n} \rightarrow+\infty$ there exists a subsequence $s_{n}$ of $t_{n}$ such that $v^{*}$ is $s_{n}$ related to $-w^{*}$.

Let there be given a sequence $t_{n} \subseteq R, t_{n} \rightarrow+\infty$. Let $v \in \Omega$ be periodic with period $\omega>0$ and choose a subsequence $s_{n}$ of $t_{n}$ such that $T_{s_{n}} v \rightarrow T_{c} v$ for some $0 \leq c \leq \omega$. Clearly $v$ is $s_{n}$ related to $T_{c} \nu$. By Proposition 4.5(3) our result will follow if we show that $\Omega^{*} \subseteq \overline{W^{s s}(v)}$ and $-\Omega^{*} \subseteq \overline{W^{u u}\left(T_{c} v\right)}$. By Lemma 4.18, $\Omega \subseteq \overline{W^{s s}(v)}$. For any vector $w \in \Omega, \overline{W^{s s}(w)} \subseteq \overline{W^{s s}(v)}$ by Proposition 3.1(7). 
Therefore, $\Omega^{*}=U_{w \in \Omega} W^{s s}(w) \subseteq \overline{W^{s s}(v)}$. Similarly, $\overline{W^{u u}\left(T_{c} v\right)}=\overline{-W^{s s}\left(-T_{c} v\right)}$ $=\overline{-W^{s s}\left(T_{-c}(-v)\right)}$ by previous facts about $W^{u u}$ and $T_{t}$. Since $T_{-c}(-v)$ is periodic, $\Omega^{*} \subseteq \overline{W^{s s}\left(T_{-c}(-v)\right)}$ by Lemma 4.18 and the argument above. Therefore $-\Omega^{*} \subseteq \overline{W^{u u}\left(T_{c} v\right)}$.

If $v \in U \cap \Omega$ then $-v \epsilon-U \cap-\Omega=-U \cap \Omega \subseteq-U \cap \Omega^{*}$. We now apply (1).

(2) Let $O^{*}, U$ be open sets in $S M$ such that $O^{*} \cap \Omega^{*} \neq \varnothing$ and $U \cap \Omega \neq \varnothing$.

(3) Let $O, U$ be open sets in $S M$ such that $O \cap \Omega \neq \varnothing$ and $U \cap \Omega \neq \varnothing$. By (2) there exists $A_{1}>0$ such that $t \geq A_{1}$ implies $T_{t}(O) \cap U \neq \varnothing$. Since $\Omega=$ $-\Omega,-O \cap \Omega \neq \varnothing$ and $-U \cap \Omega \neq \varnothing$. Using (2) again we may choose $A_{2}>0$ such that $t \geq A_{2}$ implies $\varnothing \neq T_{t}(-O) \cap(-U)=-T_{-t}(O) \cap-U=-\left\{T_{-t}(O) \cap U\right\}$. Thus $T_{-t}(O) \cap U \neq \varnothing$ for $t \geq A_{2}$. If $A=\max \left\{A_{1}, A_{2}\right\}$, then $T_{t}(O) \cap U \neq \varnothing$ for $|t| \geq A$.

Theorems 4.14 and 4.16 suggest a promising way to investigate metric problems of the geodesic flow. For example, is the geodesic flow ergodic (metrically mixing) if it is true that, for every $W^{s}$ invariant ( $W^{s s}$ invariant) set $A \subset S M$, either $A$ or $S M-A$ has measure zero with respect to the natural flow invariant measure on $S M$ ? Hedlund [11] used this method to prove that the geodesic flow on a surface of constant negative curvature and finite area is metrically mixing. See also the related papers of E. Hopf ([13], [14]).

In [6] we considered Riemannian manifolds $M$ without conjugate points such that the simply connected Riemannian cover $H$ satisfies the uniform Visibility axiom (Definition 1.6), and we showed that if $\Omega=S M$ then the geodesic flow is topologically transitive on SM. Although the law of cosines no longer holds in $H$ the uniform Visibility axiom implies those consequences of the law that are needed to prove topological transitivity on $S M$. One may define $H(\infty)$, topologize $\bar{H}=H \cup H(\infty)$, define limit sets and prove the basic results listed in $\S 1$ of this paper.

We encounter difficulties at an early stage if we try to use the method of limit spheres to prove that the geodesic flow is topologically mixing on SM if $\Omega=S M$ and if $M$ is of the type above. To define limit spheres in $H$ that possess the nice properties listed in Proposition 2.9 and the facts preceding Proposition 2.10 , it is crucial to prove that the a function of Definition 2.5 is continuous. The proof of the continuity of a depends on the fact that the functions $B_{t} \rightarrow B$ uniformly on compact subsets of $S H \times H$, and this result depends on special trigonometric properties of $H$ implied by the law of cosines. Lacking the law of cosines in $H$ it is not clear that the uniform Visibility axiom is strong enough to define limit spheres with nice properties for each point $x \in H(\infty)$. 
5. Theorems for the existence of a vector $v \in \Omega$ such that $\Omega \subseteq \overline{W^{s s}(v)}$, and the classification of such vectors.

Theorem 5.1. Let $M=H / D$ be any complete manifold with $K \leq 0$. Suppose that $\Omega$ is nonempty and that $\Omega \subseteq \overline{W^{S}(w)}$ for every vector $w \in \Omega$. Then there exists a vector $v \in \Omega$ such that $\Omega \subseteq \overline{W^{s s}(v)}$ if either of the following conditions is satisfied:

(1) There exist periodic vectors $v, v^{\prime} \in \Omega$ with periods $\omega, \omega^{\prime}$ such that $\omega / \omega^{\prime}$ is irrational.

(2) There exist periodic vectors in $\Omega$ with arbitrarily small periods.

Proof. (1) Let $v, v^{\prime}$ be periodic vectors with periods $\omega, \omega^{\prime}$ such that $\omega / \omega^{\prime}$ is irrational. We first show that $v^{\prime} \in \overline{W^{s s}(v)}$. Let $O$ be a neighborhood of $v^{\prime} \epsilon$ SM and choose $\epsilon>0$ such that $T v_{t}^{\prime} \in O$ for $-\epsilon \leq t \leq \epsilon$. For any real number $r$ let $[r]$ be the unique real number such that $0 \leq[r]<1$ and $r-[r]$ is an integer. Since $\omega / \omega^{\prime}$ is irrational the numbers $\left\{\left[n \omega / \omega^{\prime}\right]: n\right.$ is an integer $\}$ are dense in the interval $[0,1]$. By Proposition 3.1 there exists a number $0 \leq c \leq \omega$ such that $T_{c} v^{\prime} \in \overline{W^{s S}(v)}$ since $v^{\prime} \in \Omega \subseteq \overline{W^{s}(v)}$. Choose integers $a, b$ such that $\mid c / \omega^{\prime}-$ $a \omega / \omega^{\prime}-b \mid<\epsilon / \omega^{\prime}$ or $\left|c-a \omega-b \omega^{\prime}\right|<\epsilon$. If $\theta=c-a \omega-b \omega^{\prime}$ then $T_{\theta} v^{\prime} \epsilon O$ by

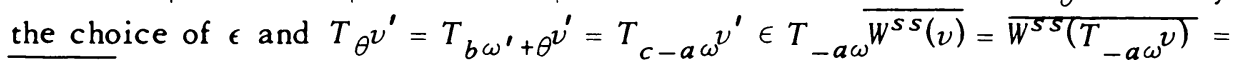
$\overline{W^{s s}(v)}$. Therefore $\overline{W^{s s}(v)} \cap O \neq \varnothing$ and $v^{\prime} \in \overline{W^{s S}(v)}$ since $O$ was arbitrary.

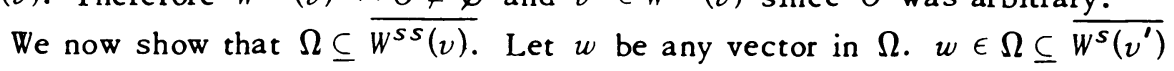
and by Proposition 3.1 there exists a number $0 \leq c^{\prime} \leq \omega^{\prime}$ such that $T_{c^{\prime}}$ w $\epsilon$ $\overline{W^{s s}\left(v^{\prime}\right)}$ or equivalently $w \in \overline{W^{s s}\left(T_{-c^{\prime}} \nu^{\prime}\right)} . T_{-c^{\prime}} v^{\prime}$ is periodic with period $\omega^{\prime}$ and

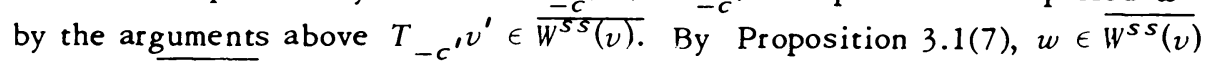
and $\Omega \subseteq \overline{W^{s s}(v)}$ since $w$ was arbitrary.

(2) Let $v \in \Omega$ be periodic with period $\omega>0$. We show that for every $t \in R$, $T_{t} v \in \overline{W^{s s}(v)}$. Let $t \in R$ be fixed and let $\epsilon>0$ be given. Let $v^{*}$ be a periodic vector with period $0<\omega^{*}<\epsilon$. By Proposition 3.1 there exists a number $0 \leq c \leq$ $\omega$ such that $T_{c} v^{*} \in \overline{W^{s s}\left(T_{-t} \nu\right)}$. Similarly there exists a number $0 \leq d \leq \omega^{*}$ such $\frac{\text { that } T}{W^{s s}(v)} d \in \overline{W^{s s}\left(T_{c} v^{*}\right)}$. Therefore $T_{d} v \in \overline{W^{s s}\left(T_{-t} v\right)}=T_{-t} \overline{W^{s s}(v)}$ or $T_{(d+t)} v \epsilon$ $\overline{W^{s s}(v)}$. Since $0 \leq d<\epsilon$ and $\epsilon$ was arbitrary, it follows that $T_{t} v \in \overline{W^{s S}(v)}$. $T_{t} v$ $\epsilon \overline{W^{s s}(v)}$ for every $t \in R$ since $t$ was arbitrary.

We show that $\Omega \subseteq \overline{W^{s s}(v)}$. Let $w$ be any vector in $\Omega$. For some number

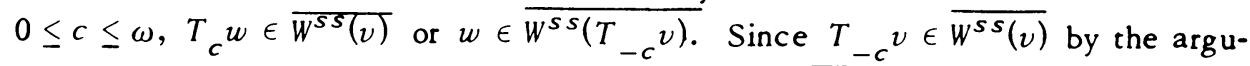
ment above it follows from Proposition 3.1 that $w \in \overline{W^{S S}(v)}$.

Theorem 5.2. Let $M=H / D$ where $H$ satisfies Axiom 1 and let $\Omega=S M$. Then there exists $v \in S M$ such that $\overline{W^{s S}(v)}=S M$.

We shall need the following 
Lemma 5.3. Let $H$ satisfy Axiom 1 and let $x \in H(\infty)$. If $f$ is any Busemann function at $x$ and if $\sigma$ is any geodesic in $H$ such that $\sigma(\infty) \neq x$ then $f(\sigma t) \rightarrow \infty$ as $t \rightarrow \infty$.

Proof. Clearly the restriction that $\sigma(\infty) \neq x$ is necessary by Proposition 2.8. Let $\sigma$ be a geodesic in $H$ such that $\sigma(\infty) \neq x$, and let $\beta$ be a geodesic in $H$ such that $\beta(-\infty)=\sigma(\infty)$ and $\beta(\infty)=x$. By Proposition $2.8 f(\beta t)=f(\beta 0)-t$ and hence $f(\beta t) \rightarrow+\infty$ as $t \rightarrow-\infty$. The geodesics $\sigma$ and $\beta^{-1}(t \rightarrow \beta(-t))$ are asymptotic. Since $f$ is convex either $f(\sigma t) \rightarrow \infty$ as $t \rightarrow \infty$ or $f(\sigma t)$ is monotone nonincreasing in $t$. By Proposition 9.8 of [4], $f(\sigma t) \rightarrow \infty$ as $t \rightarrow \infty$.

Geometrically this result means that $\gamma_{p x}$ is the only geodesic starting at $p$ that is contained in the limit ball $N(p, x)$. This property has already been noticed for the limit balls of hyperbolic space. One may show, in fact, that if this property is satisfied for all points $x \in H(\infty)$, then Axiom 1 is satisfied in $H$.

Proof of Theorem 5.2. We begin by showing that for any two open sets $O, U$ of $S M$ there exists $v \in O$ such that $W^{s s}(v) \cap U \neq \varnothing$. Let open sets $O, U$ of $S M$ be given and let $V(p, x) \in\left(\pi_{*}\right)^{-1}(O), V(q, y) \in\left(\pi_{*}\right)^{-1}(U)$. Since $\Omega=S M$ it follows from Propositions 4.9 and 4.10 that $L(D)=H(\infty)$. By Proposition 4.8, $x$ and $y$ are dual relative to $D$, and we may therefore choose a sequence $\phi_{n} \subseteq D$ such that for any point $r \in H, \phi_{n} r \rightarrow x$ and $\phi_{n}^{-1} r \rightarrow y$. It follows from Proposition 2.9 that given two points $a, b$ in $H$, if $x \in H(\infty)$ is a limit of points in $H$ equidistant from $a$ and $b$ then $L(a, x)=L(b, x)$. Such a point $x$ always exists since the equidistant set is noncompact. For each $n$ let $x_{n}$ be any point in $H(\infty)$ such that $L\left(p, x_{n}\right)=L\left(\phi_{n} q, x_{n}\right)$.

We shall establish the following facts: (1) $x_{n} \rightarrow x$, (2) $\chi_{q}\left(\phi_{n}^{-1} p, \phi_{n}^{-1} x_{n}\right)$ $\rightarrow 0$, (3) $\chi_{q}\left(\phi_{n}^{-1} x_{n}, y\right) \rightarrow 0$.

(1) Let $t \geq 0$ be fixed and, for each $n$, let $\sigma_{n}=\gamma_{p \phi_{n} q}$. For $n$ sufficiently large $t \leq d\left(p, \phi_{n} q\right)$ and we see by the convexity of the Busemann function $f_{n}$ : $r \rightarrow a\left(p, x_{n}, r\right)$ that $\alpha\left(p, x_{n}, \sigma_{n} t\right) \leq \max \left\{\alpha\left(p, x_{n}, p\right), \alpha\left(p, x_{n}, \phi_{n} q\right)\right\}=0$. Passing to a subsequence let $x_{n} \rightarrow z \in H(\infty)$. If $\sigma=\gamma_{p x}$ then $\sigma_{n} t \rightarrow \sigma t$ since $\phi_{n} q \rightarrow x$. $\alpha(p, z, \sigma t) \leq 0$ by the continuity of $\alpha$. If $f$ is the Busemann function: $r \rightarrow$ $\alpha(p, z, r)$, then $f(\sigma t) \leq 0$ for all $t \geq 0$ since $t$ was arbitrary. By the preceding lemma $x=\sigma(\infty)=z$. $x_{n}$ converges to $x$ since every convergent subsequence of $x_{n}$ converges to $x$.

(2) If $\gamma_{n}=\gamma_{p x_{n}}$, then $\phi_{n}^{-1} \circ \gamma_{n}=\gamma_{\phi_{n}^{-1} p \phi_{n}^{-1} x_{n}}$. By Remark 1.7 it suffices to show that $d\left(q, \phi_{n}^{-1} \circ \gamma_{n}\right) \rightarrow \infty$. We shall show that $d\left(q, \phi_{n}^{-1} \circ \gamma_{n}\right)=d\left(\phi_{n} q, \gamma_{n}\right)$ $\geq t_{n} / 2$ where $t_{n}=d\left(p, \phi_{n} q\right)$. If $0 \leq t \leq t_{n} / 2$ then $d\left(\phi_{n} q, \gamma_{n} t\right) \geq t_{n} / 2$ by the triangle inequality. Let $t \geq t_{n} / 2$ and let $f_{n}$ be the Busemann function: $r \rightarrow$ $a\left(p, x_{n}, r\right)$. Then $0=f_{n}\left(\phi_{n} q\right)=f_{n}\left(\gamma_{n} 0\right)$ and by Proposition 2.8,d $\left(\phi_{n} q, \gamma_{n} t\right) \geq$ $\left|f_{n}\left(\phi_{n} q\right)-f_{n}\left(\gamma_{n} t\right)\right|=\left|f_{n}\left(\gamma_{n} t\right)\right|=t \geq t_{n} / 2$. Hence $d\left(q, \phi_{n}^{-1} \circ \gamma_{n}\right)=d\left(\phi_{n} q, \gamma_{n}\right) \geq$ $t_{n} / 2$. 
(3) Since $\phi_{n}^{-1} p \rightarrow y, \chi_{q}\left(\phi_{n}^{-1} p, y\right) \rightarrow 0$. Therefore $\chi_{q}\left(\phi_{n}^{-1} x_{n}, y\right) \leq$ $\not_{q}\left(\phi_{n}^{-1} x_{n}, \phi_{n}^{-1} p\right)+\not_{q}\left(\phi_{n}^{-1} p, y\right) \rightarrow 0$.

By (3) it follows that $\phi_{n}^{-1} x_{n} \rightarrow y$ and hence $V\left(q, \phi_{n}^{-1} x_{n}\right) \rightarrow V(q, y)$. Since $x_{n} \rightarrow x$ we see that for $n$ sufficiently large $V\left(p, x_{n}\right) \in\left(\pi_{*}\right)^{-1}(O)$ and $V\left(q, \phi_{n}^{-1} x_{n}\right) \in\left(\pi_{*}\right)^{-1}(U) . V\left(\phi_{n} q, x_{n}\right)=\left(\phi_{n}\right)_{*} V\left(q, \phi_{n}^{-1} x_{n}\right) \in W^{s s}\left(V\left(p, x_{n}\right)\right)$. If $v_{n}=\pi_{*} V\left(p, x_{n}\right)$ and $w_{n}=\pi_{*} V\left(q, \phi_{n}^{-1} x_{n}\right)$, then for $n$ sufficiently large $v_{n} \in O$ and $w_{n} \in W^{s s}\left(v_{n}\right) \cap U$.

We now complete the proof of Theorem 5.2. Let $O_{n}$ be a countable basis for the topology of $S M$ and let $O$ be any open set in $S M$. By the argument above there exists $v_{1} \in O$ such that $W^{s s}\left(v_{1}\right) \cap O_{1} \neq \varnothing$. By Proposition 3.1 we can find an open set $A_{1}$ such that $v_{1} \in A_{1}$ and, for every $v \in A_{1}, W^{s s}(v) \cap O_{1} \neq \varnothing$. We may assume that $\bar{A}_{1} \subseteq O$ and $\bar{A}_{1}$ is compact. Inductively we construct a sequence of open sets $A_{i}$ such that $\bar{A}_{i} \subseteq A_{i-1}$ and a sequence $v_{i}$ of unit vectors such that $v_{i} \in A_{i}$ and, for every $\nu \in A_{i}, W^{s s}(v) \cap O_{i} \neq \varnothing$. The sets $\bar{A}_{i}$ have the finite intersection property in the compact set $\bar{A}_{1}$ and hence there exists $w \in \bigcap_{i=1}^{\infty} \bar{A}_{i}$. Since $\bar{A}_{i} \subseteq A_{i-1}, W^{s s}(w) \cap O_{i} \neq \varnothing$ for every $i$. The refore $S M$ $=\overline{W^{S S}(w) \text {. }}$

We showed in Theorem 4.14 that if $\Omega=S M$ and if $\overline{W^{s}(v)}=S M$ for some $v \epsilon$ $S M$, then $\overline{W^{S}(\nu)}=S M$ for all $v \in S M$. The corresponding statement for the sets $W^{s s}$ is false. In fact, in the case where $\Omega$ is arbitrary and $H$ satisfies Axiom 1, we classify the vectors $v \in \Omega$ such that $\Omega \subseteq \overline{W^{s s}(v)}$, assuming that such vectors exist. Hedlund obtained a partial classification in [12] for the case that $M$ is a surface of constant negative curvature such that $\Omega=S M$. See also the discussion of dense horospheres by Anosov in [1, p. 31]. As a corollary of our classification we shall show that if $M=H / D$ where $H$ satisfies Axiom 1 , then $M$ is compact if and only if $\overline{W^{S S}(v)}=S M$ for every $v \in S M$.

Definition 5.4. Let $M=H / D$ be an arbitrary complete manifold with $K \leq 0$. $A$ unit speed geodesic $\alpha$ of $M$ is almost minimizing if there exists a number $c>0$ such that $d(\alpha 0, a t) \geq t-c$ for every $t \geq 0 . v \in S M$ is almost minimizing if $\gamma_{v}$ in an almost minimizing geodesic in $M$.

Theorem 5.5. Let $M=H / D$ where $H$ satisfies $A x i o m 1$ and suppose that $\Omega \subseteq \overline{W^{s s}(v)}$ for some $v \in \Omega$. If $w \in \Omega$, then $\Omega \subseteq \overline{W^{s S}(w)}$ if and only if $w$ is not almost minimizing.

We shall need the following result, which is Proposition 7.4 of [8].

Proposition 5.6. Let $M=H / D$ be an arbitrary complete manifold with $K \leq$ 0 . Let $\alpha$ be a geodesic in $M$ and let $\beta$ be any lift of a to $H$. Then $\alpha$ is almost minimizing if and only if $\beta(\infty) \in O_{b}(D)=H(\infty)-L_{b}(D)$, the borocyclic ordinary points. 
Proof of Theorem 5.5. We first show that if $\Omega \subseteq \overline{W^{s s}(w)}$ for some $w \in \Omega$, then $w$ is not almost minimizing. Suppose that $\Omega \subseteq \overline{W^{s s}(w)}$ for $w \in \Omega$ and let $V(p, x)$ be a lift of $w$. By Proposition 5.6 we must show that $x \in L_{b}(D)$, and to do this it suffices by Proposition 2.11 to show that for any limit ball $N$ at $x$ there exists $\phi \in D$ such that $\phi p \in N$. Let $N=N(q, x)$ and $q^{\prime} \in N$ be given. Since

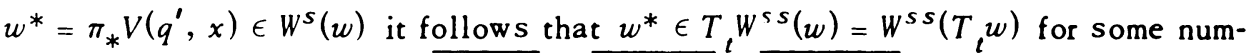
ber $t$. Hence $\Omega=T_{t} \Omega \subseteq T_{t} \overline{W^{s s}(w)}=\overline{W^{s s}\left(T_{t} w\right)}=\overline{W^{s s}\left(w^{*}\right)}$. Let $w_{n} \subseteq W^{s s}\left(w^{*}\right)$ be a sequence converging to $w$, and let $V\left(q_{n}^{\prime}, x\right)$ be a lift of $w_{n}$ such that $q_{n}^{\prime} \epsilon$ $L\left(q^{\prime}, x\right)$. Choose a sequence $\phi_{n} \subseteq D$ such that $\left(\phi_{n}\right)_{*} V\left(q_{n}^{\prime}, x\right)=V\left(\phi_{n} q_{n}^{\prime}, \phi_{n} x\right)$ $\rightarrow V(p, x)$. By Proposition 1.2, $d\left(\phi_{n} q_{n}^{\prime}, p\right)=d\left(q_{n}^{\prime}, \phi_{n}^{-1} p\right) \rightarrow 0$. If $f$ is any Busemann function at $x$ then $\left|f\left(\phi_{n}^{-1} p\right)-f\left(q^{\prime}\right)\right|=\left|f\left(\phi_{n}^{-1} p\right)-f\left(q_{n}^{\prime}\right)\right| \leq d\left(\phi_{n}^{-1} p, q_{n}^{\prime}\right) \rightarrow 0$. Since $q^{\prime} \in N, f\left(q^{\prime}\right)<f(q)$ and hence for $n$ sufficiently large $f\left(\phi_{n}^{-1} p\right)<f(q)$ or $\phi_{n}^{-1} p \in N$. Therefore $x \in L_{b}(D)$.

Let $w \in \Omega$ be not almost minimizing; we will show that $\Omega \subseteq \overline{W^{s s}(w)}$. First we will prove that there is a periodic vector $w^{*} \in \overline{W^{s s}(w)}$. Let $V(p, x)$ be a lift of $w . x \in L_{b}(D)$ by Proposition 5.6, and there exists a sequence $\phi_{n} \subseteq D$ such that $\phi_{n} p \rightarrow x$ in the horocycle topology. Passing to a subsequence, let $\phi_{n}^{-1} x$ $\rightarrow y \in H(\infty)$ in the cone topology. Since $\Omega \neq \varnothing$ there exist two distinct points in $H(\infty)$ that are dual relative to $D$ by Proposition 4.9. Since dual points lie in $L(D)$, $L(D)$ has at least two points and by Theorem 8.10 of $[8] M=H / D$ contains a closed geodesic a of period $\omega>0$. Let $\gamma$ be a lift of $a$ to $H$ and orient $\gamma$ so that $\gamma(\infty) \neq y$. Choose $\phi \in D$ such that $\phi(\gamma t)=\gamma(t+\omega)$ for every $t \in R$. If $f$ is any Busemann function at $x$ then $/\left(\phi_{n} p\right) \rightarrow-\infty$ since $\phi_{n} p \rightarrow x$ in the horocycle topology. Since $\left|f\left(\phi_{n} \gamma 0\right)-f\left(\phi_{n} p\right)\right| \leq d\left(\phi_{n} \gamma 0, \phi_{n} p\right)=d(\gamma 0, p)$ for every $n$, it follows that $f\left(\phi_{n} \gamma 0\right) \rightarrow-\infty$ and $\phi_{n} \gamma 0 \rightarrow x$ in the horocycle topology. For $n$ sufficiently large $f\left(\phi_{n} \gamma 0\right)<0$ and $\phi_{n} \gamma(\infty) \neq x$, and it follows by Lemma 5.3 that there exist numbers $t_{n}>0$ such that $\phi_{n} \gamma t_{n} \in L(p, x)$. By Proposition 2.12, $t_{n}=$ $d\left(\phi_{n} \gamma 0, \phi_{n} \gamma t_{n}\right) \geq d\left(\phi_{n} \gamma 0, L(p, x)\right) \rightarrow \infty$. Since $y \neq \gamma(\infty)$ it follows by Proposition 1.5 that $\chi_{\phi_{n} \gamma_{t_{n}}}\left(\phi_{n} \gamma 0, x\right)=\chi_{\gamma_{t_{n}}}\left(\gamma 0, \phi_{n}^{-1} x\right) \rightarrow 0$, or in other words, the angle subtended by $V\left(\phi_{n} \gamma t_{n}, x\right)$ and $V\left(\phi_{n} \gamma t_{n}, \phi_{n} \gamma 0\right) \rightarrow 0$. Choose positive integers $a_{n}$ and numbers $0 \leq c_{n} \leq \omega$ such that $t_{n}=c_{n}+a_{n} \omega$. If $d_{n}=-a_{n} \omega$ and $\psi_{n}=\phi_{n} \circ$ $\phi^{a}{ }_{n}$ then $V\left(\phi_{n} \gamma t_{n}, \phi_{n} \gamma 0\right)=\left(\psi_{n}\right)^{*} V\left(\gamma c_{n}, \gamma d_{n}\right){ }^{n}$. Passing to a subsequence $c_{n} \rightarrow c$ and $V\left(\gamma c_{n}, \gamma d_{n}^{n}\right) \stackrel{n}{\rightarrow} V(\gamma c, \gamma(-\infty))=-\gamma^{\prime}(c)$ since $d_{n} \rightarrow-\infty$. If $w_{n}=$ $\left(\psi_{n}^{-1}\right)_{*} V\left(\phi_{n} \gamma t{ }_{n}, x\right)$, then by passing to a subsequence $w_{n} \rightarrow-\gamma^{\prime}(c) . \pi_{*} w_{n} \epsilon$ $W^{s s}(w)$ since $\phi_{n} \gamma t_{n} \in L(p, x)$ and $\pi_{*}{ }^{u \prime}{ }_{n} \rightarrow-\pi_{*} \gamma^{\prime}(c)=-\alpha^{\prime}(c)$. Hence $w^{*}=$ $-\alpha^{\prime}(c) \in \overline{W^{s s}(w)}$.

Next we show that $\Omega \subseteq \overline{W^{s}\left(w^{*}\right)}$. Arguing as in the proof of Theorem 6.2 in the next section we need only show that $L(D)$ has more than two points. Choose $v \in \Omega$ such that $\Omega \subseteq \overline{W^{S S}(v)}$ and let $v^{*} \in S H$ be a lift of $v . x=\gamma_{v^{*}}(\infty) \in L(D)$ 
since $x$ and $\gamma_{\nu^{*}}(-\infty)$ are dual by Proposition 4.9. $\left(\pi_{*}\right)^{-1} \Omega \subseteq\left(\pi_{*}\right)^{-1} \overline{W^{s s}(\nu)} \subseteq$ $\overline{U_{\phi \in D} W^{s}\left(\phi_{*} v^{*}\right)}$. If $L(D)$ had at most two points then $x$ would be a common fixed point of $D$ by Theorems $8.9 \mathrm{P}$ and $8.9 \mathrm{~A}$ of [8], and we would have $\left(\pi_{*}\right)^{-1} \Omega$ $\subseteq W^{s}\left(v^{*}\right)$. Since $-v^{*} \epsilon\left(\pi_{*}\right)^{-1} \Omega-W^{s}\left(v^{*}\right) L(D)$ must have more than two points.

Since $v \in \Omega \subseteq \overline{W^{s}\left(w^{*}\right)}$ and $w^{*}$ has period $\omega>0$ there exists a number $0 \leq$ $c \leq \omega$ such that $T_{c} v \in \overline{W^{s s}\left(w^{*}\right)}$. Finally $\Omega=T_{c} \Omega \subseteq T_{c} \overline{W^{s s}(v)}=\overline{W^{s s}\left(T_{c} v\right)} \subseteq$ $\overline{W^{s S}\left(w^{*}\right)} \subseteq \overline{W^{s s}(w)}$ by Proposition 3.1(7).

6. Applications. We now apply the results of the earlier sections to Visibility manifolds $M=H / D$ ( $H$ satisfies Axiom 1$)$.

Theorem 6.1. A Visibility manifold $M$ is compact if and only if $\overline{W^{s S}(v)}=S M$ for every $v \in S M$.

Theorem 6.2. Let $M=H / D$ be a Visibility manifold. If either of the following conditions is satisfied then conclusions (1) through (3) of Theorem 4.15 are true:

(1) SM contains periodic vectors $v, v^{\prime}$ with respective periods $\omega, \omega^{\prime}$ such that $\omega / \omega^{\prime}$ is irrational.

(2) SM contains periodic vectors with arbitrarily small periods.

Theorem 6.3. Let $M=H / D$ be a Visibility manifold such that $\Omega=S M$. Then the geodesic flow is topologically mixing on SM.

Theorem 6.4. Let $M=H / D$ be a compact manifold with $K \leq 0$ such that $H$ admits no isometric, totally geodesic imbedding of the plane $R^{2}$. Then the geodesic flow is topologically mixing on SM.

Theorem 6.5. Let $S_{n}=\{$ Visibility manifolds $M=H / D$ of dimension $n \geq 2$ such that the geodesic flow is topologically mixing on SM\}. Then $S_{n}$ is closed under nontrivial normal (Galois) Riemannian coverings for each $n \geq 2$.

Proof of Theorem 6.1. If $M$ is compact then $\Omega=S M$. This follows since any compact Riemannian manifold $M$ has finite measure with respect to the natural flow invariant volume element on $S M$. By Theorem 5.2 there exists $v \in S M$ such that $\overline{W^{s S}(v)}=S M$. Since $M$ has finite diameter, $S M$ contains no almost minimizing vectors, and by Theorem 5.5, $\overline{W^{s S}(v)}=S M$ for every $v \in S M$.

We now suppose that $\overline{W^{S S}(v)}=S M$ for every $v \in S M$. First we show that $\Omega=S M$. In Theorem 4.14 the proof of $(5) \Rightarrow(3)$ did not use the hypothesis that $\Omega=S M$. Hence $D(x)$ is dense in $H(\infty)$ for every $x \in H(\infty)$. To show that $\Omega=$ $S M$ it suffices by Proposition 4.9 to show that any two points $x, y \in H(\infty)$ are dual relative to $D$. Let $x, y \in H(\infty)$ be given. Let $z$ be a point in $L(D)$, and let $z^{*}$ be a point dual to $z$. By Proposition 2.2 of [7], $z$ is dual to any point in $\overline{D\left(z^{*}\right)}=H(\infty)$. In particular $z$ is dual to $x$. Since $y \in \overline{D(z)}=H(\infty), x$ is dual to $y$ by the same argument. 
Since $\Omega=S M$, SM contains no almost minimizing vectors by Theorem 5.5. To conclude the proof it suffices to show that any noncompact, complete manifold $\dot{M}$ contains an almost minimizing vector in $S M$. If $M$ is noncompact and complete, then for any point $p \in M$ we can find a sequence $p_{n} \subseteq M$ such that $d\left(p, p_{n}\right) \rightarrow \infty$ by the Hopf-Rinow theorem. Let $\gamma_{n}$ be a shortest geodesic from $p$ to $p_{n}$ and let $v_{n}=\gamma_{n}^{\prime}(0)$. Passing to a subsequence let $v_{n} \rightarrow v \in M_{p}$. If $\gamma=\gamma_{v}$ then $d(\gamma 0, \gamma t)$ $=t$ for all $t \geq 0$, and $v$ is an almost minimizing vector.

Proof of Theorem 6.2. Because of Theorems 4.15 and 5.1 it suffices to show that $\Omega \subseteq \overline{W^{s}(v)}$ for every $v \in \Omega$. We show first that $L(D)$ has at least three points under either hypothesis (1) or (2). If $L(D)$ is a singleton, then by Proposition 8.9P of [8] there are no periodic vectors in $S M$. If $L(D)$ has only two points, then by Proposition 8.9A of [8], $D \approx \pi_{1}(M)$ is infinite cyclic and the periods of the periodic vectors in $\Omega$ are integer multiples of a smallest period. Therefore if either (1) or (2) holds then $L(D)$ has at least three points:

Let $v, w$ be any two vectors in $\Omega$ with respective lifts $v^{*}, w^{*} \in S H$. By Proposition 4.10, $\gamma_{v^{*}}(-\infty)$ and $\gamma_{w^{*}}(\infty)$ are in $L(D)$ and by Proposition 4.8 they are dual. By Proposition 4.9, $w \in P^{-}(v)$ and since $v, w$ were arbitrary $\Omega \subseteq$ $P^{-}(v)$ for every $v \in \Omega$. If $v \in \Omega$ then $\overline{W^{s}(v)}=P^{-}(v)$ and therefore $\Omega \subseteq \overline{W^{s}(v)}$ for every $v \in \Omega$.

Condition (1) is very likely to be satisfied for a Visibility manifold $M=H / D$ such that $\Omega \neq \varnothing$ and $\pi_{1}(M)$ is not infinite cyclic. By Theorem 8.10 of [8], $M$ contains infinitely many distinct "inequivalent" closed geodesics.

Proof of Theorem 6.3. By Theorem 8.10 of [8], SM contains a periodic vector, and by Theorem 5.2 there exists $v \in S M$ such that $\overline{W^{S S}(v)}=S M$. The result now follows from Theorem 4.16.

Proof of Theorem 6.4. By Theorem 4.1 of [6] if $M=H / D$ is a compact manifold with $K \leq 0$, then $H$ satisfies Axiom 1 if and only if $H$ admits no isometric, totally geodesic imbedding of the plane $R^{2}$. The result now follows from Theorem 6.3.

Proof of Theorem 6.5. It follows from Theorem 6.3 and the equivalent definitions of topological transitivity that for a Visibility manifold $M=H / D$ the condition $\Omega=S M$ is equivalent to the conditions that the geodesic flow be topologically mixing or topologically transitive on SM. From Proposition 4.10 it follows that, for a Visibility manifold, $\Omega=S M$ if and only if $L(D)=H(\infty)$. Given $n \geq 2$, let $M=H / D \in S_{n}$ and let $M^{\prime}$ be any normal (Galois) covering of $M . M^{\prime}$ $=H / N$ where $N$ is a normal subgroup of $D$. By Proposition 9.12 of [8] and the discussion above $L(N)=L(D)=H(\infty)$. Therefore $\Omega=S M^{\prime}$ and $M^{\prime} \in S_{n}$ by Theorem 6.3.

Remarks. (1) In Theorem 6.5 the set $S_{n}$ contains many noncompact manifolds of infinite volume. For example, if $M$ is a compact orientable surface of 
genus $k \geq 2$ then $M$ admits a metric with $K \equiv-1$. If $M^{\prime}$ is the normal covering surface whose fundamental group is the commutator subgroup of $\pi_{1}(M)$, then $M^{\prime}$ has infinite area. $M$ and $M^{\prime}$ are in $S_{2}$.

(2) Let $M=H / D$ be a complete manifold with $K \leq 0$ whose geodesic flow on $S M$ is topologically transitive. The topological transitivity condition is a very strong one as is shown by Theorem 4.14, among other things. It seems very likely that $H$ must satisfy Axiom 1, at least if $M$ is compact.

\section{BIBLIOGRAPHY}

1. D. V. Anosov, Geodesic flows on closed Riemannian manifolds of negative curvature, Trudy Mat. Inst. Steklov. $90(1967)$ = Proc. Stelov Inst. Math. 90 (1967). MR 36 $\# 7157$.

2. N. P. Bhatia and G. P. Szegö, Dynamical systems: stability theory and applications, Lecture Notes in Math., no. 35, Springer-Verlag, Berlin, 1967, p. 122. MR 36 \#2917.

3. G. D. Birkhoff, Dynamical systems, Amer. Math. Soc. Colloq. Publ., vol. 9, Amer. Math. Soc., Providence, R. I., 1927.

4. R. Bi shop and B. O'Neill, Manifolds of negative curvature, Trans. Amer. Math. Soc. 145 (1969), 1-49. MR $40 \# 4891$.

5. H. Busemann, The geometry of geodesics, Academic Press, New York, 1955. MR 17, 779 .

6. P. Eberlein, Geodesic flow in certain manifolds without conjugate points, Trans. Amer. Math. Soc. 167 (1972), 151-170.

7. - Geodesic flows on negatively curved manifolds. I, Ann. of Math. (2) 95 (i972), 492-510.

8. P. Eberlein and B. O'Neill, Visibility manifolds, Pacific. J. Math. (to appear).

9. A. Grant, Surfaces of negative curvature and permanent regional transitivity, Duke Math. J. 5 (1939), 207-229.

10. G. Hedlund, The dynamics of geodesic flows, Bull. Amer. Math. Soc. 45 (1939), $241-260$.

11. - Fuchsian groups and mixtures, Ann. of Math. 40 (1939), 370-383.

12. - Fuchsian groups and transitive horocycles, Duke Math. J. 2 (1936), 530542.

13. E. Hopf, Statistik der geodätischen Linien in Mannigfaltigkeiten negativer Krümmung, Ber. Verh. Sächs. Akad. Wiss. Leipzig 91 (1939), 261-304. MR 1, 243.

14. - Statistik der Lösungen geodäitscher Probleme vom unstabilen Typus II, Math. Ann. 117 (1940), 590-608. MR 2, 106.

15. B. O'Neill, (to appear).

DEPARTMENT OF MATHEMATICS, UNIVERSITY OF CALIFORNIA, LOS ANGELES, CALIFORNIA 90024

DEPARTMENT OF MATHEMATICS, UNIVERSITY OF CALIFORNIA, BERKELEY, CALIFORNIA 94720 